\title{
Erfolgreich Präsentieren in der Wissenschaft? Empirische Untersuchungen zur kommunikativen und kognitiven Wirkung von Präsentationen
}

\author{
MALGORZATA DYNKOWSKA, HENNING LOBIN \\ und VERA ERMAKOVA
}

\section{Abstract}

In multimodal scholarly presentations supported by presentation software, spoken and written language, various visualizations on the projected slides as well as the contributors' gestures and facial expressions build a meaningful oneness. On the one hand, communication scientists as well as linguists have for a relatively long time neglectedthe presentation as a complex form of communication. On the other hand, since Tufte (2003), columnists of major German newspapers have been dealing with the question of the value, the quality and the place of Power Point in science, they have even tried to find the answer to the question whether Power Point is evil or not.

The presentation practice is perceived as fundamentally deficient of systematic empirical research on presentations. Also Grabowski called attention to this desideratum with two critical articles (Grabowski 2003, 2008).

Various questions - still unanswered - have motivated the implementation of a number of experiments (in the summer of 2010) for analyzing the knowledge and learning effects and the communicational impact of scientific presentations. The general aim of these experiments was to conduct empirical research on selected presentations in order to find out what kind of presentation is successful. The main interest is to find out which model of scholarly presentation produces the best results regarding learning effect and communicative impact.

\section{Einführung}

Schätzungen zufolge benutzen heute 400 Millionen Menschen pro Tag weltweit das am weitesten verbreitete Präsentationsprogramm Powerpoint (Ross 2008). Computergestützte Präsentationen, die mit Unterstützung von Powerpoint oder mit einer alternativen Präsentationssoftware gehalten werden, sind nahezu allgegenwärtig. In der Wissenschaft setzten sie sich in den letzten zwei Jahrzehnten als Kommunikationsform durch, blieben als Untersuchungsgegenstand aber insbesondere von Kommuni- 
kationsforschern und Linguisten relativ lange unbeachtet. Feuilletonisten großer deutscher Tageszeitungen beschäftigen sich dagegen bereits seit 2003, d. h. seit der Erscheinung der zwei viel zitierten Veröffentlichungen von Edward Tufte, kontinuierlich mit der Frage, ob Powerpoint „böse" sei und beantworten sie zumeist mit einem klaren „Ja“ (Schlaffer 2004; Becker 2005; Dambeck 2006; Joffe 2007; Steinfeld 2009).

Die Präsentationspraxis wurde und wird auch aktuell grundsätzlich als defizitär wahrgenommen. Von diversen Qualitätsmängeln bleibt auch der Wissenschaftsbereich nicht verschont, worauf der Linguist Grabowski in seinen präsentationskritischen Artikeln aufmerksam macht (Grabowski 2003, 2008). Dieser Umstand darf aus zwei Gründen überraschen: Zum einen etablierten sich computergestützte Präsentationen als Kommunikationsform doch in nahezu allen Bereichen des gesellschaftlichen Lebens und in der Wissenschaft; zum anderen haben wir - parallel zur Etablierung von Präsentationen - mit einer bis heute andauernden Konjunktur an Ratgeberliteratur gepaart mit unterschiedlichen Trainingsformaten zu tun.

Inzwischen liegt neben den unzähligen Präsentationsratgebern eine Reihe von wissenschaftlichen Publikationen vor, die Präsentationen ${ }^{1}$ aus verschiedenen Perspektiven betrachten. Dabei sehen die WissenschaftlerInnen die im Kontext der „Powerpoint ist böse“-Debatte gemachten weitgreifenden Äußerungen relativ skeptisch und weisen vor allem auf eine fehlende empirische Untermauerung der Aussagen hin (siehe z. B. Lobin 2009a; Bucher et al. 2010). Systematische empirische Untersuchungen zur Rezeption von Powerpoint-Präsentationen fehlen weitgehend.

Bisherige Studien zu Einsatzmöglichkeiten multimodaler Wissensvermittlung und zur Wirkung von Powerpoint-Präsentationen - vorwiegend psychologischer oder didaktischer Provenienz - erbrachten eher indifferente Ergebnisse. Sie fragten dabei vor allem danach, $o b$ der Einsatz von Powerpoint Verbesserungen in der Vermittlung von Inhalten bewirkt. Die Studien fragten aber nicht, wie dies bei der Verwendung von Powerpoint optimal zu geschehen hat. Diese bis dato unbeantwortete Forschungsfrage motivierte die Realisierung einer Reihe von Rezeptionsexperimenten zur Wissensvermittlung und zum Kommunikationserfolg unterschiedlicher wissenschaftlicher Präsentationen ${ }^{2}$. Der vorliegende Beitrag präsentiert in erster Linie Ergebnisse dieser Experimente (Abschnitt 5). Die Darstellung der Ergebnisse wird dabei vor dem Hintergrund der Vielfalt und Disparität der Untersuchungsergebnisse, Meinungen und des bestehenden Erfahrungswissens über die PowerpointPräsentationen und ihre Rezeption vorgenommen (Abschnitt 2). Abschnitt 3 skizziert eine neuartige Betrachtungsweise, die die komplexe multimodale Struktur von Präsentationen mit linguistischem Instrumen- 
tarium zu beschreiben und zu analysieren erlaubt. Dieser linguistische Ansatz bildete den theoretischen Hintergrund, auf dem das Untersuchungsdesign der Gießener Experimentalstudie entwickelt wurde (Abschnitte 4 und 5). Der Beitrag schließt mit einer Interpretation der Ergebnisse ab (Abschnitt 6).

\section{Das (wissenschaftliche) Interesse an Powerpoint}

Der Einsatz von Powerpoint-Projektionen zur Begleitung eines Vortrages und die große Verbreitung der neuen Kommunikationsform Präsentation sorgten für ein großes Interesse vorwiegend aufgrund der vielen negativen Schlagzeilen, die das Programm seit 2003 in den USA und seit Anfang 2004 in Deutschland machte. Die so genannte „Powerpoint ist böse"-Debatte lebt auch immer wieder auf ${ }^{3}$, zuletzt Ende 2009 mit der in der Süddeutschen Zeitung von Thomas Steinfeld gestellten Frage „Ist Powerpoint gefährlich?" und einer sehr pauschalen Verurteilung der Verwendung von Powerpoint in der Wissenschaft (Steinfeld 2009). Diesmal lagen allerdings bereits einige wissenschaftliche Untersuchungsergebnisse zu wissenschaftlichen Präsentationen vor, die schnell plausible Entgegnungen ermöglichten (z. B. Lobin 2009b; Peters 2009). Denn viele Angriffe gegen das Programm Powerpoint und somit gegen die Kommunikationsform der Präsentation beruhen auf persönlichen Beobachtungen und Erfahrungen der Kritiker und konzentrieren sich vorwiegend auf die Folienprojektion. So werfen die Wissenschaftler den Powerpoint-Kritikern auch vor allem mangelnde empirisch gesicherte Basis und eine weitgehend fehlende „qualitative Bewertung der Teilelemente einer Präsentation oder eine Beurteilung ihres Zusammenspiels" (Bieber 2009: 140) vor. Aus wissenschaftlicher bzw. linguistischer Sicht ist eben zunächst eine systematische Betrachtung der Funktionsweise aller medialen Konstituenten der hochkomplexen Kommunikationsform Präsentation (hier Modalitäten genannt, siehe Abschnitt 3) notwendig, um Aussagen über ihren Kommunikationserfolg anhand von Befunden aus empirischen Untersuchungen machen zu können.

Erste Untersuchungen zu Präsentationen, die deren Kommunikationserfolg skeptisch einschätzen, etwa Tufte (2003a, 2003b), berücksichtigen nicht alle Spezifika der Kommunikationsform, sondern verabsolutieren die Eigenschaften des Präsentationsprogramms oder eine als defizitär wahrgenommene Praxis (Shwom und Keller 2003; Zillien 2005, 2006).

Dass die gegenwärtig zu beobachtende Präsentationspraxis in der Wirtschaft und in der Wissenschaft immer noch kritisch bewertet werden kann und soll, ist unumstritten (siehe etwa Grabowski 2003). Die mangelhafte Qualität der inzwischen etablierten Kommunikationsform dürfte insofern überraschen, als der Buchhandel unzählige Ratgeber zur 
Verfügung stellt. Diese lassen sich in drei Kategorien einteilen: i) allgemeine Ideenratgeber mit Empfehlungen zur Präsentationsvorbereitung, -durchführung und -nachbereitung; ii) Ratgeber zum Umgang mit Präsentationssoftware und iii) Mischformen, in denen einerseits allgemeine Empfehlungen für eine erfolgreiche Präsentation ausgesprochen und andererseits Anleitungen zur Nutzung von Präsentationssoftware (vorwiegend Powerpoint) gegeben werden ${ }^{4}$. Die nahezu unüberschaubare und recht heterogene, dennoch immer noch blühende Landschaft der Ratgeberliteratur verweist auf ein grundsätzliches Problem (siehe auch Schnettler et al. 2007; Lobin 2009a): Die wenigsten Empfehlungen sind durch Daten und Erkenntnisse zur Güte und Wirkung von Präsentationen fundiert; eine nähere Betrachtung entpuppt zuweilen widersprüchliche Aussagen. Der Hinweis auf u. a. den Wissenschaftler Clifford Stoll und seine persönlichen Beobachtungen zu Powerpoint-Präsentationen (Stoll 2002: 202 ff.) in einem der aktuellen Ratgeber (Danz 2010) verleiht der Publikation keineswegs eine notwendige wissenschaftliche Begründung. Keins der Ratgeber bietet darüber hinaus eine systematische integrative Sicht auf die komplexe Kommunikationsform Präsentation und ihre drei Modalitäten (siehe Abschnitt 3), die begründete Ratschläge im Sinne einer praktischen Präsentationsrhetorik erlauben würde (vgl. Lobin 2009a: 118 ff. $)^{5}$.

Die hier sehr kurz skizzierte Problematik lässt unmittelbar zwei Fragen aufkommen: Wie werden wissenschaftliche Präsentationen nun tatsächlich rezipiert? Was sind die Erfolgskriterien beim Präsentieren? In den folgenden Abschnitten soll die Forschungsszenerie zum Thema (wissenschaftliche) Präsentationen und erste Ansätze zur Beantwortung der beiden Fragen kurz vorgestellt werden.

Mit grundlegenden Aspekten von Präsentationen im Allgemeinen beschäftigen sich erstmals zusammenfassend Schnettler und Knoblauch (2007). Die thematisch breit gefasste Diskussion in diesem Sammelband, der größtenteils Ergebnisse eines Forschungsprojektes zu computergestützten Präsentationen vermittelt, umfasst ausgewählte theoretische Aspekte (u. a. Knape 2007; Peters 2007; Lobin 2007) sowie erste empirische Befunde zur Erforschung „,visuell unterstützter Präsentationen“ als einer neuen kommunikativen Form bzw. kommunikativen Gattung. Ein sehr breites Spektrum unterschiedlicher video-graphisch erhobener Präsentationsformen aus u. a. Vorstandssitzungen, Mitarbeiterschulungen, Produktdemonstrationen, Selbstdarstellungen von Firmen und Fachkonferenzen bildet den Gegenstand für die Analysen von Detailaspekten. So untersuchen Schnettler (2007) und Knoblauch (2007) das Verhältnis von gesprochener Sprache und visueller Projektion mit einem konversationsanalytischen Methodeninstrumentarium. Besonders werden dabei deiktische Gesten fokussiert. Aus ihrer Analyse im Präsentationszusammen- 
hang wird die Position abgeleitet, dass in Präsentationen in ihrem Vollzug erst die eigentlichen Kommunikationsobjekte erzeugt werden. Eine ähnliche Position in Bezug auf sprachliche Deixis vertritt (Brinkschulte 2007). In weiteren Beiträgen werden Kriterien zur Klassifikation (Pötzsch 2007), Evaluation (König 2007) und Gattungsvarianz (Yates und Orlikowski 2007) entwickelt. Im Kontext der Eingangsfrage nach dem (Rezept für den) Kommunikationserfolg von Powerpoint-Präsentationen scheint die Untersuchung von König interessant: Mit einer Fragebogenuntersuchung und vertiefenden Interviews hat sie „Ethnokategorien' ermittelt, die der Bewertung der Präsentationsgüte zugrunde liegen (z. B. einheitliches Layout, sparsamer Einsatz von Animationen etc.). Fraglich ist allerdings, ob die Befragten vor allem Kriterien wiedergegeben haben, die sie inzwischen aus den Hunderten von Ratgebern aufgenommen haben, und ob eben diese Ratgeber solche eher konventionellen Erwartungen wiederum durch ihre Empfehlungen verstärken. Nicht behandelt in den Studien in Schnettler und Knoblauch (2007) werden u. a. Fragen nach Prozessen der Kohärenzbildung sowie spezifischen Rezeptionsmustern in den Präsentationen, die im Hinblick auf ihre genuine Eigenschaft der Multimodalität und im Kontext ihrer Basisfunktion der Wissensvermittlung - hochinteressante und relevante Ergebnisse zur Beantwortung der eingangs gestellten Fragen liefern könnten.

Eine erneute theoretische Auseinandersetzung mit Präsentationen nimmt das zwei Jahre später erschienene Sammelband von Coy und Pias (2009) vor und situiert die immer wieder im feuilletonistischen Diskurs gestellte Frage, ob Powerpoint böse sei, im Spannungsfeld von Informatik, Medien-, Kunst- und Kulturwissenschaften. Die Beiträge gehen dabei von „einer elementaren Bindung von Wissen und Erkenntnis an Verfahren und Formen der Darstellung" aus; die Experten beschäftigen sich allerdings jeweils mit anderen Aspekten der Powerpoint-Präsentationen, etwa mit einer Rekonstruktion „des medialen Eigensinns“ von Powerpoint u. a. anhand seiner technischen Vorgeschichte. Coy und Pias stellen am Anfang fest, „[d]ie Frustration durch schlechte Darbietungen wird eher von schlechten Inhalten als von schlechter Präsentationstechnik verursacht [...]." (Coy und Pias 2009: 10). Dennoch fordern viele Autoren technische und ästhetische Kompetenz im Präsentationsalltag (u. a. Rebensburg 2009), derer Vermittlung empirische Präsentationsforschung vorausgehen müsste (Bieber 2009). Deren Befunde könnten und sollten in eine empirisch begründete Präsentationsrhetorik einfließen, welche z. B. Pflüger (2009) als notwendig ansieht.

Die Kommunikationsform (wissenschaftliche) Präsentation kann wissenschaftlich aus unterschiedlichen Perspektiven betrachtet werden, wie in den vorausgehenden Abschnitten skizziert. Inzwischen liegen auch soziologische (Schnettler und Knoblauch 2007; Knoblauch 2009), linguisti- 
sche (Lobin 2009a; Lobin et al. 2010; Fricke 2008), medien- (Bucher et al. 2010; Niemann und Krieg 2011) und theaterwissenschaftliche (Peters 2011) Ansätze vor, die einen theoretischen Hintergrund für empirische Untersuchungen abgeben ${ }^{6}$. Arbeiten didaktischer Provenienz zu Präsentationen mit Unterstützung von Präsentationssoftware konzentrieren sich, wie Adams (2008) konstatiert, , auf praktische Ratgeberliteratur sowie auf gelegentliche Einsätze im Unterricht“ (ebd., 7). Psychologische und didaktische Forschungsansätze fragen vorwiegend nach Funktion, Wirkung und Einsatzmöglichkeiten multimodaler Wissensvermittlung in und mit Präsentationen. Empirische Studien wurden dabei vorwiegend in den USA durchgeführt und untersuchten auf der Grundlage ähnlicher methodischer Designs die Auswirkungen der Verwendung von Präsentationstechnologie in der universitären Lehre: Lehrveranstaltungen wurden abwechselnd mit und ohne Präsentationen durchgeführt (oft als direkter Vergleich zwischen Overhead- und PPT-Präsentationen) und abschließend von Studierenden bewertet. Ergebnisse derartiger Untersuchungen bringen ein disparates Bild: Es werden entweder positive Effekte auf den Lernprozess (Lowry 1999; Frey und Birnbaum 2002; Earnest 2006) ermittelt oder keine signifikante Verbesserung der Arbeitsleistungen konstatiert (Szabo und Hastings 2000; Rankin und Haas 2001; Bartsch und Cobern 2003). Die Hypothese, dass die simultane Vermittlung von Informationen über mehrere Sinneskanäle das Verstehen und Behalten der Inhalte bei den Rezipienten erleichtert, konnte also nicht eindeutig belegt werden. In vielen Befragungen wurde allerdings eine grundsätzlich positive Einstellung der Studierenden gegenüber dem Einsatz von Präsentationssoftware in den Lehrveranstaltungen festgestellt. Diese Überzeugung wird in einer der späteren Befragungen nicht bestätigt: Young (2004) berichtet über eine beachtliche Unzufriedenheit von Undergraduate-Studenten mit der Verwendung von Powerpoint durch Dozenten.

Einige der vorhandenen Arbeiten zu Powerpoint nehmen Bezug auf bestehende Ergebnisse kognitionspsychologischer Befunde zur Rezeption multimodaler Darstellungen und versuchen diese auf die Rezeption von Powerpoint-Präsentationen zu übertragen (etwa Müller-Prove 2009). Untersuchungen zur Rezeption multimodaler Darstellungen in instruktionalen Kontexten legen dabei nahe, dass simple Summierungsannahmen - je mehr Modi, desto besser der Rezeptionserfolg - nicht tragen (z. B. Sweller 2002). Der Einsatz von statischen oder animierten Visualisierungen oder deren Kombination in multimodalen Formen führen nicht automatisch zu einer besseren Behaltensleistung, sondern können das Lernverhalten negativ beeinflussen und Überforderungen erzeugen. Dies bestätigte die an der University of North Carolina Wilmington durchgeführte Studie, in der Auswirkungen von zwei „Powerpoint lectu- 
res" mit und ohne benutzerdefinierte Animationen auf die Wissensleistungen der Rezipienten untersucht wurden. In beiden Szenarien wurden allerdings keine klassischen Powerpoint-Präsentationen mit Rednern eingesetzt, sondern Folienprojektionen mit ,voice-over"-Kommentaren. Zur Funktion der Animationen heißt es: „The incremental introduction of concepts in dynamic slides was designed to prevent student exhaustion caused by visually presenting all concepts at once (ebd.)." Die AutorInnen konstatieren: "Subjects show the static slides had better recall of graphics and text on the slides due to prolonged exposure to the information (Mahar et al. 2009: 588)." Ergebnisse der Studie stimmen laut Mahar et al. mit Befunden anderer früherer Untersuchungen überein?

Einen durchaus positiven Einfluss von Animationen auf die Steuerung von Aufmerksamkeit im Rezeptionsprozess ermittelte dagegen die an der Universität Trier realisierte Rezeptionsstudie zu wissenschaftlichen Vorträgen mit Powerpoint-Projektionen, die die Methode der Blickaufzeichnung nutzte. Die Trierer Medienwissenschaftler identifizierten Animationen und Einblendungen „als effektives Steuerungselement für die Aufmerksamkeitsverteilung von Rezipienten“ (Niemann und Krieg 2011), was auch den Aussagen der wahrnehmungspsychologischen SalienzTheorie entspricht. Dynamische Folienelemente bedingen bei der Rezeption einen Wechsel zwischen dem Gesagten und dem Gezeigten (alternierendes Rezeptionsmuster). Wird in der Präsentation dagegen auf Animationen und auf kohärenzsichernde Maßnahmen verzichtet, finde eine „kontinuierliche Verzahnung von mündlichem Vortrag und Folientext bei der Rezeption" nicht statt. Zu fragen wäre an dieser Stelle, ob eine Verzahnung der Rede und der projizierten Folien, d. h. das alternierende Rezeptionsmuster, für das Verstehen der vermittelten Inhalte und ihre Memorierbarkeit förderlich ist.

Eine empirische Präsentationsforschung steht noch am Anfang, dennoch können bereits erste empirische Befunde einige der Kritikpunkte an der Software Powerpoint und den von ihr begleiteten Präsentationen widerlegen. So zeigen beispielsweise Blickdaten von Rezipienten in der Trierer Rezeptionsstudie, die als Indikatoren für Aufmerksamkeitsallokation während einer Präsentation ausgelegt werden, dass ,[e]ine Konzeption der Folien im Hinblick auf Unterstuitzung des Vortrags [...] sogar dazu beitragen [kann], dass die Aufmerksamkeit für den mündlichen Vortrag zunimmt" (Bucher et al. 2010: 403). Somit wäre der Vorwurf, dass projizierte (Powerpoint-)Folien die Aufmerksamkeit des Publikums (und des Präsentators selbst) gänzlich auf sich ziehen, entkräftet. Festzuhalten ist allerdings, dass trotz erster empirischer Ansätze kaum gesicherte Erkenntnisse zu Mechanismen der Rezeption und zur kommunikativen Wirkung von wissenschaftlichen Präsentationen vorliegen; es überwiegen immer noch Hypothesen zu ihrem Kommunikationserfolg, 
die insbesondere in der öffentlichen Debatte verbreitet und diskutiert wurden. Angesichts der bestehenden weitgehend defizitären Forschungslage wurden am Gießener Zentrum für Medien und Interaktivität Rezeptionsexperimente realisiert, in denen erstmals systematisch die Auswirkungen verschiedener Präsentationsszenarien bzw. Präsentationsstile empirisch untersucht wurden (siehe Abschnitt 4).

Bevor die Ergebnisse der Gießener Experimente zur Präsentationsqualität dargestellt werden, soll im folgenden Abschnitt zunächst eine linguistisch motivierte Betrachtungsweise der multimodalen Struktur von Präsentationen skizziert werden. Diese wurde anhand von linguistischen Analysen realer wissenschaftlicher Präsentationen entwickelt (ausführlich siehe Lobin et al. 2010).

\section{Zur Struktur und Funktionsweise wissenschaftlicher Präsentationen}

Wie bereits eingangs erwähnt, basiert das Untersuchungsdesign der in Gießen realisierten Experimente zur Kommunikationsqualität wissenschaftlicher Präsentationen (siehe Abschnitt 4) auf einer linguistisch begründeten Modellierung der Funktionsweise wissenschaftlicher Präsentationen als einer multimodalen Kommunikationsform. Die Grundlage dieser Modellierung bilden Befunde aus einer linguistischen Analyse von 33 realen Präsentationen dreier Wissenschaftskulturen (Geistes-, Naturund Wirtschaftswissenschaften), die im Kontext wissenschaftlicher Veranstaltungen, Tagungen, Konferenzen etc. stattgefunden haben und mithilfe von Präsentationssoftware unterstützt wurden ${ }^{8}$. Im Folgenden soll der theoretische Rahmen kurz vorgestellt werden. Die Ausführungen basieren auf der Annahme, dass Präsentationen als multimodale Texte verstanden werden können und somit bestimmte wiederkehrende Eigenschaften aufweisen, die zum einen ihre Struktur betreffen und zum anderen sich auf den kommunikativen Zusammenhang beziehen, in dem sie vorkommen (ausführlich siehe Lobin et al. 2010; Lobin 2009a).

Die grundlegende Eigenschaft wissenschaftlicher Präsentationen ist ihre Multimodalität, d.h. eine simultane Ko-Präsenz unterschiedlicher kommunikativer Elemente wie gesprochener und geschriebener Sprache, Gestik und Mimik des Präsentators, diverser Visualisierungen auf den projizierten Folien sowie Film- und Tondokumenten ${ }^{9}$. In der Aufführungsphase werden Präsentationen in einer spezifischen Kombination dieser Elemente konstituiert. Für analytische Zwecke kann man die Elemente drei Modalitäten zuordnen: der sprachlichen (im Sinne von gesprochener Sprache), der visuellen und der performativen Modalität ${ }^{10}$.

Die sprachliche Modalität bilden alle Elemente des mündlichen Vortrags, das heißt der Rede des Präsentators. Als gesprochener Text weist die Rede Eigenschaften der gesprochenen Sprache auf; zugleich ist sie 
als wissenschaftlicher Text häufig durch konzeptionelle Schriftlichkeit geprägt. Auch die projizierten Folien beinhalten natürlich sprachliche bzw. textuelle Elemente. Da diese visuell vermittelt werden, werden sie im Kontext der visuellen Modalität behandelt (siehe unten). Die Spezifik der wissenschaftlichen Kommunikation, die bestimmte Anforderungen unter anderem hinsichtlich der Präzision der Formulierung und der Darstellung der Inhalte an die wissensehaftliche Textproduktion stellt, bedingt eine prinzipiell hohe Intensität bei der Vorbereitung bzw. Inszenierung der Rede. In der Präsentationspraxis ist die Rede jedoch in unterschiedlichem Maße vorbereitet. So wurde in den geistes- (GW) und wirtschaftswissenschaftlichen (WW) Präsentationen des ausgewerteten Korpus vorwiegend frei vorgetragen (GW: $73 \%$; WW: 72\%). In den naturwissenschaftlichen Präsentationen gibt es eine geringe Dominanz des sog. gemischten Vortragsstils (abwechselnd freier Vortrag und Ablesen: $54 \%$ ). In den natur- und wirtschaftswissenschaftlichen Präsentationen wurde darüber hinaus grundsätzlich nicht von einem Vortragsmanuskript abgelesen. In den geisteswissenschaftlichen Vorträgen kommen zwar Sequenzen vor, in denen verlesen wird, diese bilden allerdings lediglich 1\% der Vortragsdauer aller Präsentationen dieses Wissenschaftsbereichs.

Die visuelle Modalität manifestiert sich grundsätzlich an der Projektionswand als Projektion von Präsentationsfolien, auf denen - wie bereits erwähnt - textuelle Elemente, aber auch Grafiken, Bilder und Videosequenzen in einer sequenziellen Abfolge übertragen werden (vgl. Abb. 1). Eine einzelne Folie und ihre Projektion kann als eine analytische Gliederungseinheit der Präsentation aufgefasst werden. Sie wird gewöhnlich durch einen markierten Folienübergang deutlich von der nächsten abgegrenzt und kann damit eine Gliederungseinheit über die visuelle Ebene hinweg darstellen. In den 33 aufgezeichneten Präsentationen wurden zu 44\% Textfolien, zu 39\% Bildfolien und zu 17\% Mischfolien (mit Textund Bild-Anteilen) verwendet. Darunter sind sowohl Inhaltsfolien subsumiert, die direkt das Thema der Präsentation betreffen, als auch sog. Rahmungsfolien, d.h. zum Beispiel Titel- und Gliederungsfolien. Die letzteren sind im Korpus zu 13\% vorhanden. 5\% der gesamten Projektionszeit in den Präsentationen fällt auf die Einblendung dieser Folien. Nicht thematisiert in den Präsentationen wurden 0,2\% der Folien; sie wurden entweder übersprungen oder inhaltlich nicht behandelt ${ }^{11}$. Hinsichtlich der Folienverwendung sind darüber hinaus Unterschiede in den drei verschiedenen Wissenschaftsbereichen zu verzeichnen: In den Präsentationen aus dem wirtschaftswissenschaftlichen Bereich dominierten eindeutig textuelle Folien (61\%). Die Textfolien wurden von den Geistesund den NaturwissenschaftlerInnen mit einer deutlich geringeren, aber vergleichbaren Häufigkeit verwendet (39\% und 36\%). Der Gesamtanteil 


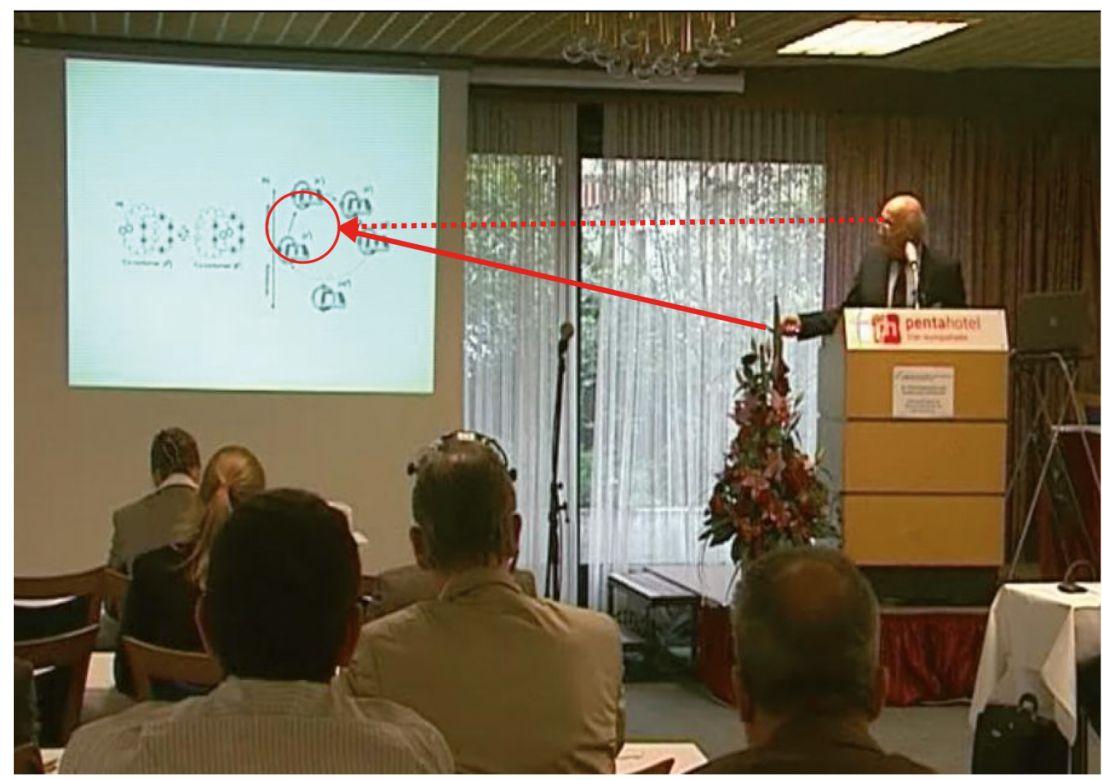

Abbildung 1. Spezifische Deixis: Zeigegeste mit Laserpointer an der Projektionswand (vgl. Lobin et al. 2010: 366).

an reinen Bildfolien und Folien mit Kombinationen aus bildlichen und textuellen Elementen (sog. Mischfolien) ist bei den Geisteswissenschaften relativ hoch und liegt bei $61 \%$, wobei den Bildfolien $45 \%$ und Mischfolien $16 \%$ zukommt $^{12}$. Was die Verwendung von Bildern oder bildlichen Elementen angeht, liegen jedoch Präsentationen aus den Naturwissenschaften vorne mit insgesamt $64 \%$ entsprechender Folien; hier ist auch eine Dominanz der Mischfolien im Vergleich zu den anderen Wissenschaftskulturen deutlich (23\%). Folienanimationen, d. h. wahrnehmbarer sukzessiver Aufbau der Präsentationsfolien, kommen am häufigsten in geistes- und nur selten in wirtschaftswissenschaftlichen Präsentationen vor $(43 \% \mathrm{zu} 17 \%)$.

Wenn man von der gesamten Präsentationsaufführung die sprachliche Modalität (Rede) und die visuelle Modalität (Folienprojektion) abzieht, so bleibt zunächst nicht viel mehr als das körperliche Verhalten des Präsentators auf der „Präsentationsbühne“ übrig. Was gehört nun zur performativen Modalität in den komplexen, multimodalen Präsentationen? In der linguistischen Forschung werden sowohl „Aspekte der Körperlichkeit“ in der mündlichen Kommunikation (u. a. Gestik) als auch die „Materialität" in der schriftlichen Kommunikation, beispielsweise Handschrift, Typografie bzw. Textdesign, als Phänomene der Text-Performanz 
betrachtet (siehe Antos 2009: 407; vgl. Bucher 2007). Können bzw. sollen Zeigehandlungen des Präsentators mit dem Textdesign der Folien mehr oder weniger additiv zu einer performativen Dimension zusammengefasst werden? ${ }^{13}$ Diese Elemente werden, wie oben verdeutlicht, in den wissenschaftlichen Präsentationen miteinander kombiniert. Einen für unsere Zwecke fruchtbaren Zugang zum Verständnis der performativen Dimension von Präsentationen eröffnet die Theatertheorie (siehe Lobin 2009a: 31 ff.), insbesondere die Anwendung der Konzepte der Inszenierung, Aufführung und der Performativität nach Fischer-Lichte (2005). Bezogen auf wissenschaftliche Präsentationen umfasst die performative Modalität demnach all das, was (erst) in der Präsentationsaufführung manifest wird, d.h.: 1) das körperbezogene Verhalten des Präsentators, zum Beispiel die Körperstellung zwischen dem Publikum und der Projektionswand, 2) zeichenhafte Bewegungen wie redebegleitende Gestik, 3) die Blickrichtung und diverse andere Formen gestischen Zeigens mit oder ohne Zeigeinstrumenten (siehe Abb. 1), 4) die Ko-Präsenz von Präsentator und Rezipienten und deren Interaktion sowie 5) bestimmte Arten von Animationen oder die Dynamisierung der Folienabfolge, die vom Redner ausgelöst werden. Die performative Modalität nutzt alle drei Raumdimensionen sowie die zeitliche Dimension. In der Performanz sind in gewissem Sinne auch die Dimensionen integriert, in denen sich die beiden anderen Modalitäten entfalten. Man kann daher die Performanz als eine Grundlage für die Verknüpfung der anderen Modalitäten betrachten. Im Folgenden soll die performative Modalität im Hinblick auf ihre integrierende Leistung fokussiert werden.

Ein wichtiges Element dieser Modalität bildet gestisches Zeigen, d. h. performative deiktische Elemente. In den Präsentationen begegnet man unterschiedlichen Formen der performativen deiktischen Handlungen. Ein Vorschlag zur Systematisierung modalitätsübergreifender deiktischer Elemente in wissenschaftlichen Präsentationen wurde in Lobin et al. (2010) vorgestellt. Dieser berücksichtigt auch sprachliche Deixis, denn in wissenschaftichen Präsentationen wird üblicherweise nicht nur mit gestischen, sondern auch mit sprachlichen Mitteln auf die visuell dargebotenen Daten verwiesen. So kann man grundsätzlich zwischen spezifischer Deixis (beispielsweise direkte Zeigegesten an der Projektionswand oder sprachliche Verweismittel) und unspezifischer Deixis unterscheiden. Bei den unspezifischen deiktischen Elementen werden direkte unspezifische (etwa allgemeine Gesten in Richtung der Projektionswand) und indirekte unspezifische Deixis (beispielsweise Zeigegesten auf den Präsentationslaptop) differenziert (ebd.). Anhand dieser Systematisierung modalitätsübergreifender deiktischer Elemente wurden die 33 realen wissenschaftlichen Präsentationen des Korpus kodiert: Es zeigte sich zunächst ein relativ homogenes Bild, d. h. eine relativ gleichmäßige Verwendung deik- 
tischer Elemente in den Präsentationen des Korpus (37\% direkte unspezifische Deixis, 33\% spezifische Deixis und 30\% indirekte unspezifische Deixis). Beim Vergleich der drei Wissenschaftskulturen kamen allerdings fachspezifische Unterschiede in Bezug auf die Formen gestischen Zeigens zutage. In den geisteswissenschaftlichen Präsentationen dominiert beispielsweise „Blick auf den Präsentationslaptop“ (42\%), in den naturwissenschaftlichen überwiegen „direkte Zeigegesten an der Projektions-

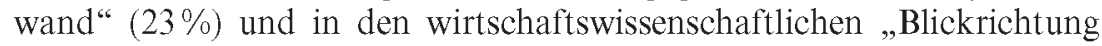
zur Projektionswand“ (37\%).

Die multimodale Analyse wissenschafticher Präsentationen geht nicht davon aus, dass die drei Modalitäten (die sprachliche, visuelle und die performative Modalität) rein additiv zur Gesamtbedeutung der Präsentationen beitragen. Vielmehr verbinden sich die einzelnen Modalitäten in ähnlicher Weise zu einer Gesamtbedeutung, wie es auf der Satzebene durch die Verbindung einzelner Wörter und die situative und diskursive Einbettung des Satzes geschieht. Dadurch entsteht auch eine sehr komplexe, mehrdimensionale Form der Multimodalität: Sie entfaltet sich in drei Raumdimensionen und einer Zeitdimension (im Vergleich zur Multimodalität zweidimensionaler Dokumente). $\mathrm{Zu}$ der Linearität im zeitlichen Verlauf des mündlichen Vortrags kommt die mit der Multimodalität per se verbundene Multi-Linearität hinzu. Es entsteht eine mehrschichtige Struktur, die sowohl eine sequenzielle als auch eine räumliche Ordnung (Cluster) aufweist. Für die Rezipienten einer Präsentationsaufführung gilt es, die Zusammenhänge zwischen allen Elementen zu erkennen und zu erfassen (vgl. Bucher et al. 2010). Wie multimodales Verstehen komplexer wissenschaftlicher Präsentationen auf der Rezipientenseite aufgebaut wird, zeigen erste empirische Befunde aus der Trierer Rezeptionsstudie mit der Blickaufzeichnungsmethode (siehe Abschnitt 2). Die Gießener Rezeptionsexperimente kennzeichnet ein anderes Untersuchungsdesign, mit dessen Hilfe erstmals die Frage nach den Auswirkungen verschiedener Präsentationsstile sowie ihrer Kommunikationsqualität beantwortet werden sollte. Den beiden Studien gemeinsam ist allerdings ein erweitertes Verständnis von Präsentationen als multimodalen Kommunikationsformen. Im folgenden Abschnitt soll das methodische Vorgehen in der Gießener Studie vorgestellt werden.

\section{Gießener Rezeptionsexperimente}

\subsection{Zielsetzung}

Die in Gießen im Juni 2010 realisierten Rezeptionsexperimente wurden mit dem Ziel durchgeführt, Erkenntnisse über die Art und Weise einer optimalen Vermittlung von Inhalten in Präsentationen zu erhalten. Unter 
einer erfolgreichen Präsentation wird dabei eine Präsentationsform verstanden, die eine hohe Kommunikationsqualität in Bezug auf die Effektivität der Informationsvermittlung und die Einschätzung des persönlichen Gesamteindrucks der Rezipienten aufweist.

Das Design der Experimente war darauf ausgerichtet, unter kontrollierten Bedingungen Rezeptionsdaten einer Präsentation zu erheben, in der nur eine oder sehr wenige Parameter in acht unterschiedlichen Szenarien systematisch variiert wurden. Folgende Fragestellungen waren dabei zentral:

- Wie wirken sich verschiedene Varianten der Verteilung und Verzahnung der Information in Rede und Folienprojektion auf die Wissensleistungen der Rezipienten aus?

- Wie wird die kommunikative Wirkung der verschiedenen Varianten der Verteilung und Verzahnung der Information in Rede und Projektion von den Rezipienten bewertet?

\subsection{Untersuchungsdesign}

Für die Zwecke der Experimentalstudie wurden mehrere Präsentationsszenarien entwickelt und getestet. Die Grundlage für die verschiedenen Varianten bildete ein speziell konzipiertes Vortragsmanuskript zum Thema „Grundlagen des Semantic Web", das auch die sprachliche Modalität (Rede) in allen Szenarien konstituierte ${ }^{14}$. Der Vortrag sollte sich an Studierende aller Fachbereiche ohne bzw. mit möglichst geringen thematischen Vorkenntnissen richten. Folgende Anpassungen des konzeptionell schriftlichen Manuskripts an die Kommunikationssituation einer mündlichen Präsentation und die Adressatengruppe wurden im Wesentlichen vorgenommen: (i) Reduzierung der Wissensvoraussetzungen für die Vortragsrezeption durch eine gezielte Verwendung von Fachbegriffen mit entsprechender Erläuterung, (ii) Verwendung anschaulicher Beispiele, (iii) vorzugsweise Verwendung einfacher Satzstrukturen sowie (iv) Kennzeichnung des thematischen Aufbaus des Vortrags (fünf Teilthemen) durch verbale Markierungen thematischer Übergänge.

Auf der Basis des Vortragsmanuskripts wurden folgende acht Präsentationsszenarien entwickelt:

1. ,Vortrag' (ohne Unterstützung mit Präsentationssoftware),

2. Vortrag mit klassischen ,Textfolien",

3. Vortrag mit ,Bildfolien",

4. Vortrag mit paraphrasierenden Folien (Szenario „Paraphrasierung'),

5. Vortrag mit Folien, die in sehr hohem Maße auf Elemente der Rede rekurrieren (Szenario, Identität'), 
6. Vortrag mit Text-Bild-Folien, in dem die sprachliche und visuelle Modalität sehr eng verzahnt werden (Szenario, enge Verzahnung'),

7. Vortrag mit Text-Bild-Folien ohne sprachliche oder performative Mittel der Verzahnung und ohne Aufmerksamkeitssteuerung (Szenario ,reduzierte Verzahnung'),

8. ,Folienprojektion“ (ohne Redner).

Die Szenarien ,Vortrag und ,Folienprojektion'stellen zwei Extreme dar; sie sind keine multimodalen Präsentationen im eigentlichen Sinne (siehe Abschnitt 2), wurden aber dennoch im Untersuchungsdesign als wichtige Bezugspunkte berücksichtigt. Kennzeichnend für den ,Vortrag' ist die sprachliche Modalität (Rede) mit körperlicher Präsenz des Redners, für das Szenario ,Folienprojektion' ausschließlich die visuelle Modalität. In der ,Folienprojektion" wurde eine Sequenz aus Präsentationsfolien an die Projektionswand kontrolliert eingeblendet. Verwendet wurde hier die Foliendatei aus dem Szenario, enge Verzahnung, daher waren die Projektionsschritte in diesem Szenario eine wichtige Orientierungsgröße für die zeitliche Festlegung der einzelnen Projektionen in dem Szenario ohne Redner.

Wissenschaftliche Präsentationen mit Unterstützung von Präsentationssoftware sind, wie oben gezeigt, eine sehr komplexe multimodale Kommunikationsform. Begründete Aussagen über Parameter, die eine optimale Wissensvermittlung und einen hohen Kommunikationserfolg beim Präsentieren garantieren können, setzen daher empirische Erhebungen zur Wirkungsweise einzelner oder nur sehr weniger Parameter voraus, die systematisch variiert werden. So wurde beispielsweise in den drei Szenarien Vortrag mit klassischen ,Textfolien', Vortrag mit ,paraphrasierenden Folien' und Vortrag mit Textfolien mit hoher Rekurrenzidentität (,Identität') vor allem die Wirkung unterschiedlich realisierter, visuell vermittelter textueller Elemente in der Präsentationssituation untersucht. Bedingt durch eine gezielte Parametervariation entstanden darüber hinaus zum einen eher untypische Präsentationsvarianten, etwa die Szenarien ,Identität ${ }^{6}$ und ,Paraphrasierung ${ }^{6}$. Zum anderen bildeten sich gegensätzliche Szenarien-Paare heraus, beispielsweise:

- Vortrag mit klassischen ,Textfolien' vs. Vortrag mit „Bildfolien“,

- das Szenario ,enge Verzahnung" vs. ,reduzierte Verzahnung",

- ,Vortrag' vs. ,Folienprojektion“ (siehe Abb. 2).

Die Variante ,Folienprojektion' kann einerseits als ein Gegenpol zu der Variante ,Vortrag' (siehe oben) angesehen werden. Andererseits bildet sie ein Ergebnis konsequenter Parameterreduzierung, die bereits in der Variante ,reduzierte Verzahnung' im Vergleich zur ,engen Verzahnung 

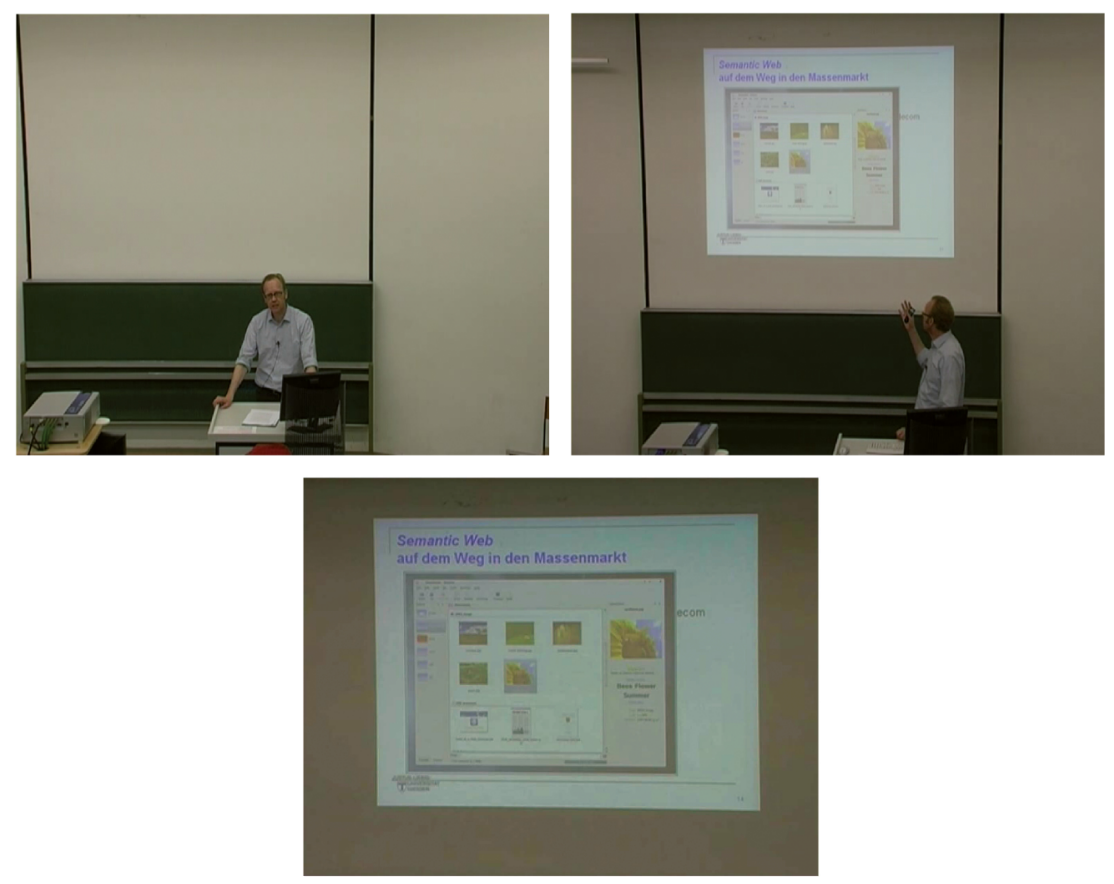

Abbildung 2. Szenarien im Vergleich (v. l.): ,Vortrag', ,enge Verzahnung' und, Folienprojektion:

angestrebt wurde. Elemente der performativen Modalität wurden in der ,reduzierten Verzahnung' auf das Geringste beschränkt (Körperpräsenz des Redners) und in der Variante ,Folienprojektion" vollständig entfernt (kein Redner). In diesem Sinne weisen die entwickelten Szenarien untereinander interessante Querbezüge auf, ohne dass Polarität vorliegt (siehe oben ,Identität' und ,Paraphrasierung').

Um vor allem einige Unterschiede zwischen den Szenarien kurz zu veranschaulichen, sollen exemplarisch Powerpoint-Folien zu derselben Redesequenz aus den Szenarien ,Identität', ,Paraphrasierung' und ,Bildfolien' gezeigt und an einem Beispielsatz erläutert werden (siehe Abb. 3). Der vom Redner gegen Ende unserer Textsequenz geäußerte Satz Das eröffnet auch eine neue Dimension der Verschmelzung von Daten aus unterschiedlichen Quellen, sogenannte Mashups wurde in den drei Szenarien unterschiedlich wiederaufgenommen:

- im Szenario ,Identität‘ wurde das lexikalische Material aus der Rede auf den Folien in der Äußerung Mashups = Verschmelzung von Daten 


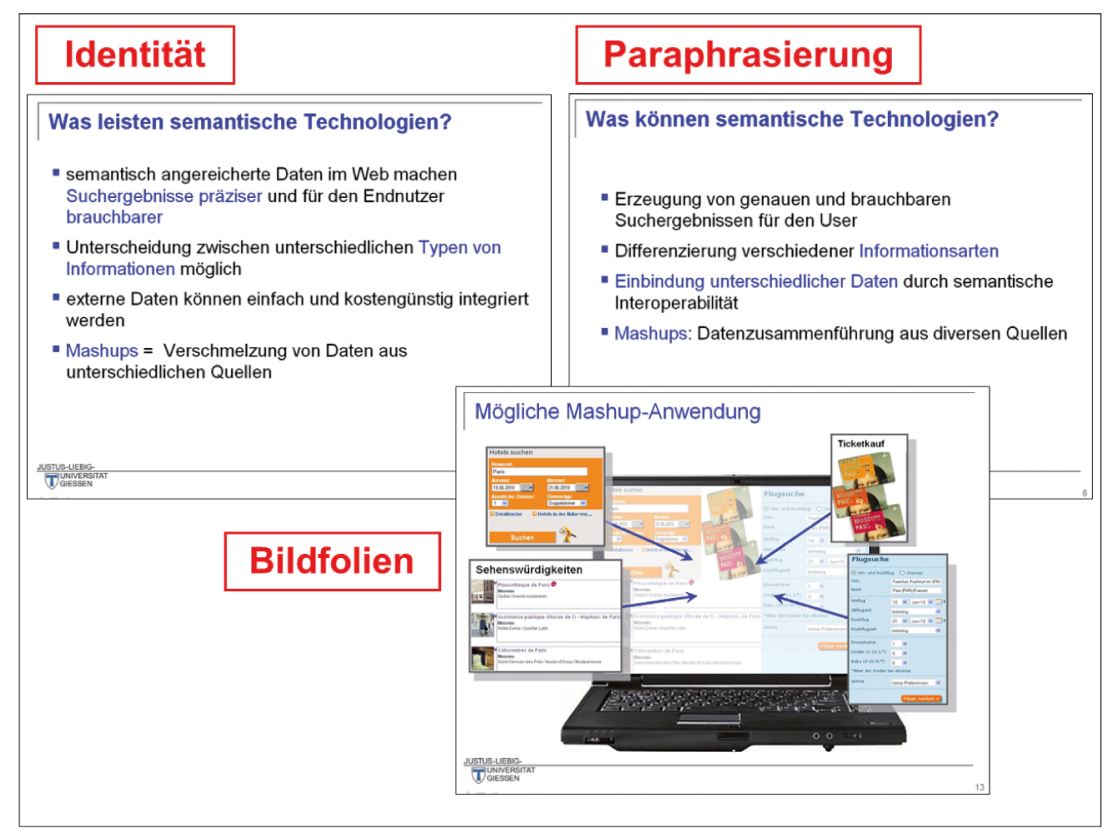

Abbildung 3. Powerpoint-Folien der Szenarien ,Identität', ,Paraphrasierung' und ,Bildfolien" im Vergleich.

aus unterschiedlichen Quellen wiederaufgenommen, d. h. es lag identische Rekurrenz von Wortfolgen vor;

- im Szenario ,Paraphrasierung dagegen wurde das vom Redner Gesagte auf den Folien paraphrasiert mit Mashups: Datenzusammenführung aus diversen Quellen, was eine höhere Verarbeitungsleistung von den Rezipienten abverlangt, so die Hypothese;

- im Szenario ,Bildfolien“ wurde das Vorgetragene mit Bildern und Grafiken illustriert oder visualisiert; für unsere Textsequenz wurde eine Visualisierung des Mashups-Beispiels gezeigt (siehe Abb. 3).

Abschließend soll auf das Design der Präsentationsvariante ,enge Verzahnung' kurz eingegangen werden, da sie als ein Prototyp multimodaler wissenschaftlicher Präsentationen aufgefasst werden kann. Konstitutiv für die Variante waren alle drei Modalitäten (siehe Abschnitt 3). Charakteristisch war dabei eine enge Verzahnung der sprachlichen und der visuellen Modalität, d.h. der Rede und der Folienprojektion (siehe Abb. 2 oben rechts). Dafür wurden bestimmte Mittel gezielt und kontrolliert eingesetzt, und zwar: 
- performative deiktische Handlungen des Präsentators (Körperhalbstellung, Zeigegesten mit Pointer und Ablesen von der Projektionswand),

- sprachliche Deixis wie auf dieser Folie,

- Rekurrenz, d.h. Wiederaufnahme sprachlicher Elemente aus der Rede auf den Folien,

- Animationen bzw. schrittweiser Aufbau von Folien.

Mit der gezielten intensiven Modellierung der Verknüpfung sprachlicher, visueller und performativer Modalität in diesem Szenario war unter anderem die Frage nach dem Einfluss der Performanz des Präsentators auf die Informationsvermittlung und den Kommunikationserfolg in wissenschaftlichen Präsentationen verbunden - ein rhetorischer Aspekt, der bei der Untersuchung wissenschaftlicher Konstitutionsprozesse bislang nur eine geringe Rolle gespielt hat.

\subsection{Setting und Durchführung}

Alle acht Präsentationsszenarien (siehe Abschnitt 4.2) wurden vor jeweils vergleichbar zusammengesetzten Gruppen von je ca. 25 Versuchsperso-

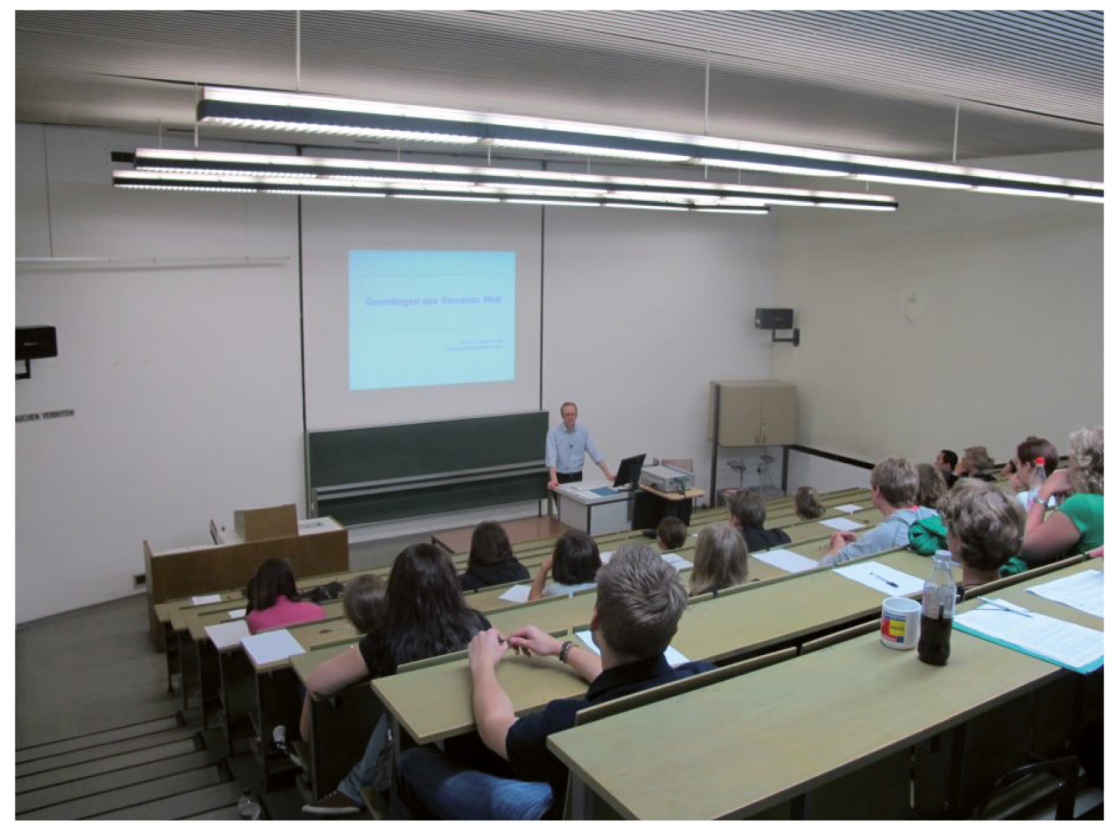

Abbildung 4. Blick in den Vorlesungssaal während eines der Rezeptionsexperimente. 


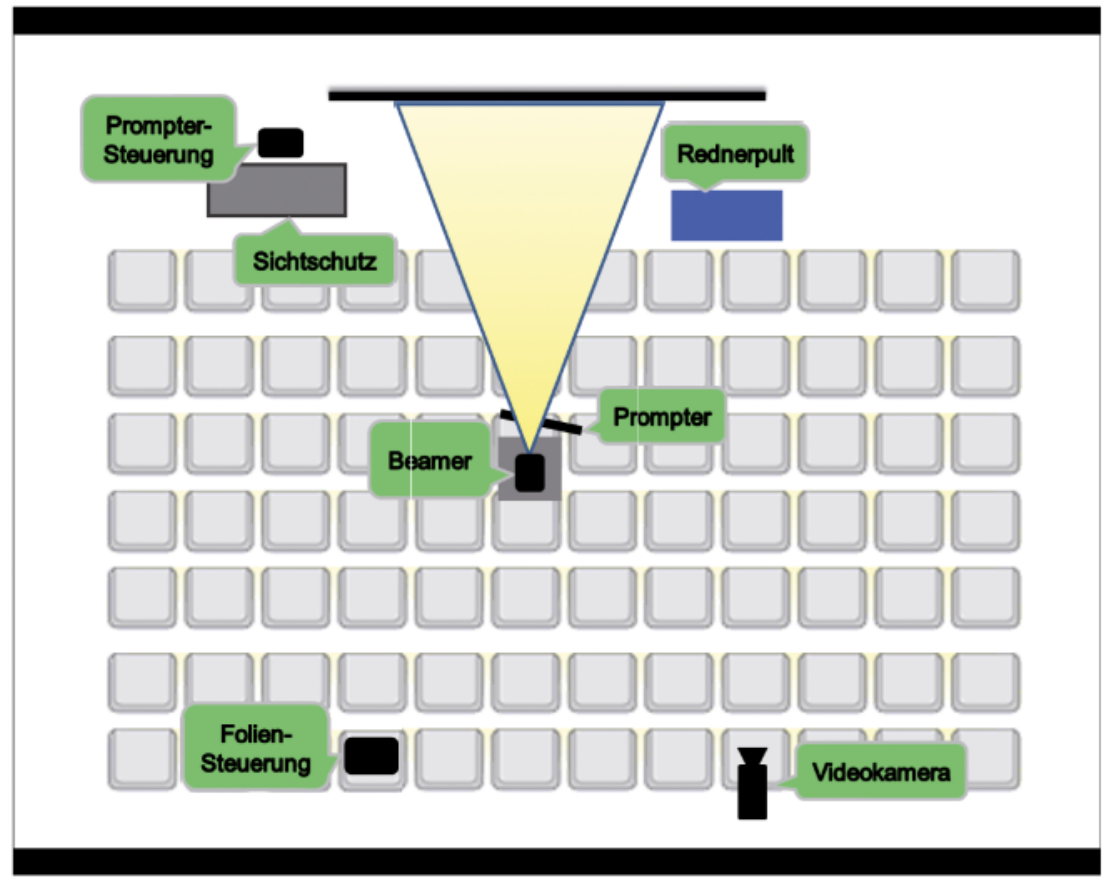

Abbildung 5. Technischer Aufbau der Experimente.

nen aufgeführt, die aus der Studentenschaft der Justus-Liebig-Universität Gießen rekrutiert wurden. Alle Aufführungen wurden in sehr kontrollierter Weise unter identischen Rahmenbedingungen durchgeführt, u. a. in demselben Vorlesungssaal mit entsprechender technischer Ausstattung, mit ein und demselben Redner in derselben Kleidung. Abb. 4 stellt ein Foto dar, das während eines Experiments gemacht wurde.

Um größtmögliche Kontrolle über die sprachliche Modalität zu erlangen, die in allen Präsentationsvarianten vorzugsweise als ,freie Rede inszeniert werden sollte, wurde ein Teleprompter eingesetzt.

Die Folien des Teleprompters beinhalteten dabei nicht nur den Text des Vortragsmanuskripts, sondern auch entsprechende Anweisungen zum Rednerverhalten. Der Redner steuerte per Fernbedienung nicht die Folienprojektion, sondern den Prompter. Den Rezipienten gegenüber vermittelte er jedoch den Eindruck, als ob die Projektion der Folien in seiner Hand liegen würde - wie es nahezu ausnahmslos in der Präsentationspraxis der Fall ist. Die Steuerung der Folienprojektion übernahm in Wirklichkeit eine geschulte Mitarbeiterin, die sich in der letzten Sitzreihe im Raum positionierte (siehe Abb. 5). Der Zeitpunkt jedes Folien- 
übergangs wurde im Untersuchungsdesign präzise festgelegt und in einer detaillierten Anweisung festgehalten. Die Skizze in Abb. 5 zeigt den vollständigen technischen Aufbau der Experimente.

Jedes der acht Experimente hatte einen identischen Ablauf (Dauer jeweils ca. 35 Minuten): Zu Beginn wurden die Testpersonen in einem Pretest-Fragebogen unter anderem $\mathrm{zu}$ thematischem Vorwissen und Wahrnehmungstyp befragt. Dann folgte eine Präsentation nach festgelegtem Szenario, die jeweils etwa 10 Minuten dauerte. Abschließend füllten die Probanden einen Posttest-Fragebogen zur kommunikativen Wirkung und einen Wissenstest aus.

\subsection{Testpersonen}

Bei der Konstruktion der acht Stichproben (siehe Abschnitt 4.2) wurde auf eine möglichst homogene Verteilung der Testpersonen in Bezug auf das Geschlecht, die fachliche Zuordnung der Studierenden sowie ihr thematisches Vorwissen geachtet. An den Experimenten nahmen insgesamt 197 Studierende teil, dabei deutlich mehr weibliche als männliche Probanden (79\% zu 21\%). Das Durchschnittsalter lag bei 23 Jahren; der Mittelwert der Angaben zum aktuellen Semester betrug 5. Was die Zuordnung zu Fachkulturen angeht, so ergab sich eine Dominanz von Studierenden geisteswissenschaftlicher Fächer.

Ihr Vorwissen in Bezug auf das Vortragsthema, d.h. Grundlagen des Semantic Web, schätzten die Rezipienten in der Pretest-Befragung grundsätzlich homogen als schlecht oder sehr schlecht ein. Wesentlich besser bewerteten sie ihre Kenntnisse im Umgang mit dem Programm Powerpoint ${ }^{15}$. Ebenso wichtig wie die Frage nach dem thematisch relevanten Vorwissen war die Frage nach dem Wahrnehmungstyp der Rezipienten, da Inhalte des Vortrags über zwei Kanäle kommuniziert werden sollten. Laut Selbsteinschätzung der Testpersonen dominierte insgesamt der „(eher) visuelle Wahrnehmungstyp“ in allen getesteten Szenarien, wobei der höchste Anteil im Szenario ,Identität ( $96 \%$ ) und der geringste in der ,Folienprojektion“ (75\%) verzeichnet wurde.

\section{Kommunikationsqualität wissenschaftlicher Präsentationen}

In den folgenden Abschnitten werden zentrale Ergebnisse und Befunde aus den 2010 in Gießen realisierten Experimenten zur Kommunikationsqualität unterschiedlicher Präsentationsstile präsentiert.

\subsection{Vermittlung von Inhalten}

Der Wissenstest, den die Testpersonen nach jeder der acht Präsentationen auszufüllen hatten, bestand aus acht Multiple-Choice-Fragen mit Mehr- 


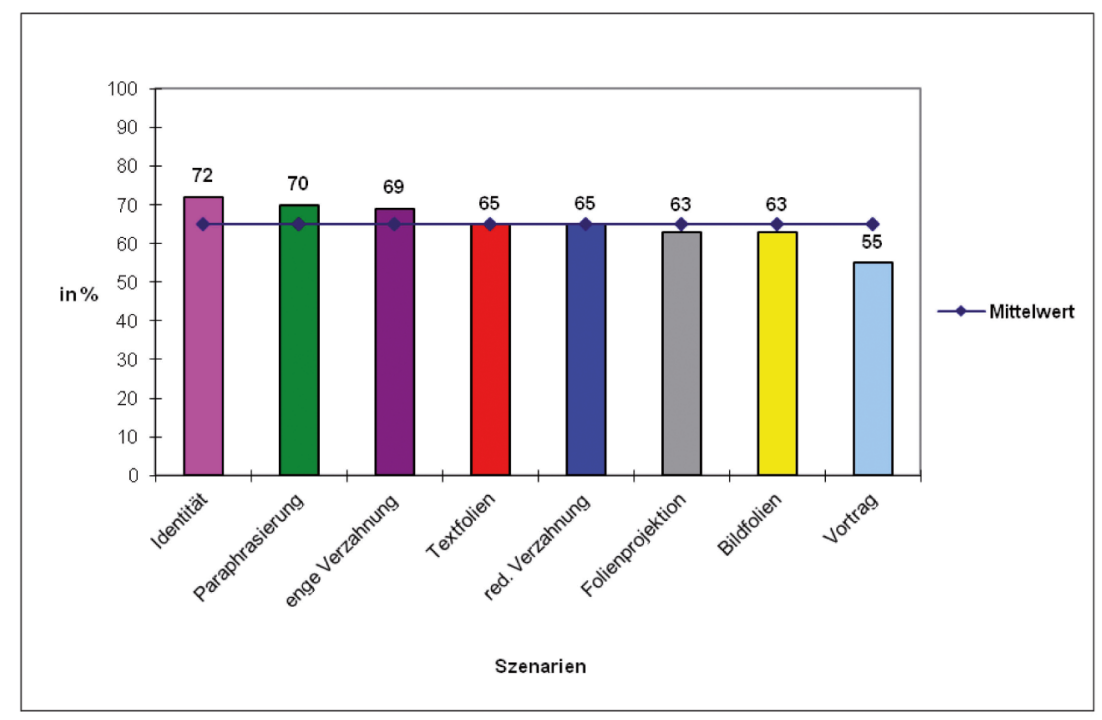

Abbildung 6. Prozentuale Auswertung des Wissenstests in allen Präsentationsszenarien.

fachantworten und einer offenen Frage. Insgesamt konnten neun Punkte bei dem Test erreicht werden. Der Test fällt insgesamt relativ gut aus: Die Mittelwerte in den einzelnen Szenarien liegen zwischen 4,9 und 6,4 Punkten (vgl. prozentuale Auswertung in Abbildung 6). Die Unterschiede in der Effektivität der Wissensvermittlung zwischen den einzelnen Szenarien sind auf den ersten Blick nicht wesentlich ${ }^{16}$. Sie sind aber keinesfalls marginal, insbesondere wenn man den höchsten in einer Präsentationsvariante erzielten Wert dem niedrigsten gegenüberstellt, d.h. $72 \%$ vs. 55\% (Abb. 6).

Am besten bewältigen den Test die Rezipienten des Szenarios ,Identität ${ }^{6}(72 \%)$, dicht gefolgt von denen aus den Szenarien ,Paraphrasierung (70\%) und ,enge Verzahnung $(69 \%)$. Dabei erzielen männliche Probanden durchschnittlich etwas bessere Ergebnisse als weibliche $(68 \% \mathrm{zu}$ $65 \%$ ). In der Geschlechterfrage gibt es allerdings keine erkennbare Tendenz, so beantworten die Wissensfragen in den Varianten ,Identität ${ }^{6}$ und ,Paraphrasierung' die Männer besser und in den Varianten ,enge Verzahnung', ,Folienprojektion' und ,Vortrag' die Frauen. Tendenziell besser lösen den Test die Probanden, die auch ihr thematisches Vorwissen als besser einschätzten (vgl. Abschnitt 4.4).

Die Szenarien ,Identität‘ und ,Paraphrasierung' wurden als Gegenpole konzipiert (siehe Abschnitt 4.2), schneiden jedoch beide ähnlich gut und im Szenarienvergleich am besten - ab. Die einzelnen Fragen des 


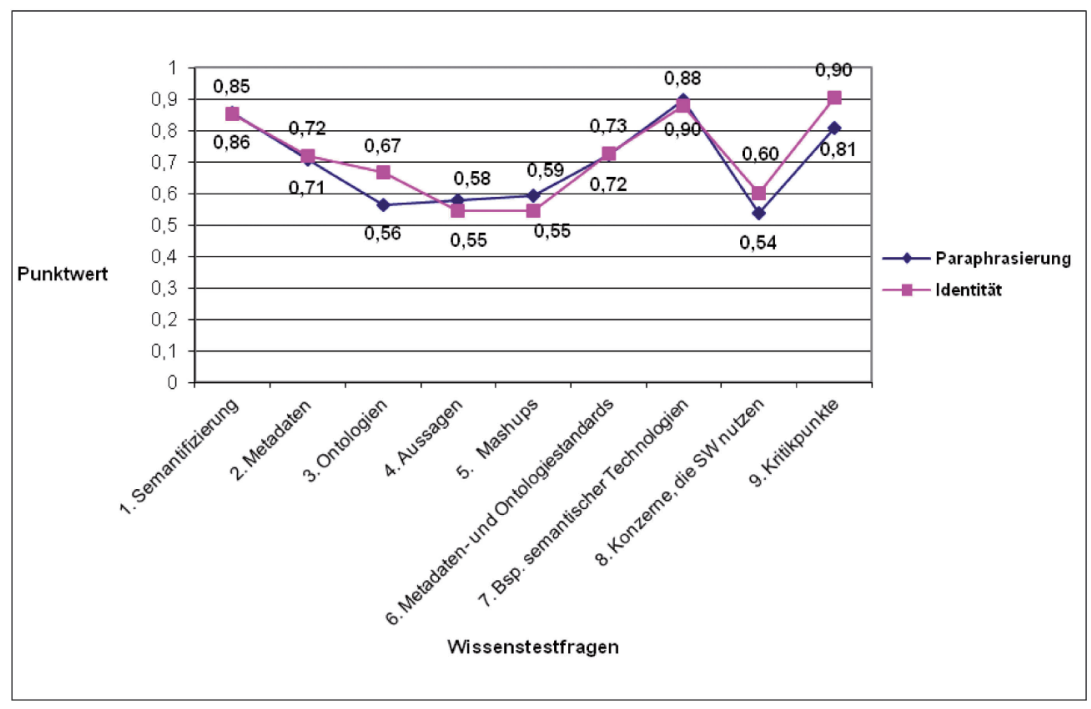

Abbildung 7. Fragenbeantwortung im Wissenstest in den Szenarien, Paraphrasierung* und, Identität'.

Wissenstests werden in diesen Szenarien auch durchgehend gut bis sehr gut beantwortet, wie in der Abb. $7 \mathrm{zu}$ sehen ist. Lediglich in Bezug auf drei Fragen (Frage 3, 8 und 9) fallen die Antworten der Probanden im Szenario ,Paraphrasierung' schlechter aus.

Schaut man sich ein weiteres gegensätzliches Szenarienpaar an, und zwar die Varianten ,Textfolien' und ,Bildfolien', so schneiden sie ebenfalls vergleichbar gut ab (65\% zu 63\%, siehe Abb. 6). Vergleicht man jedoch das Szenario ,Bildfolien " mit den Szenarien, in denen reine Textfolien oder Mischfolien verwendet wurden, so erbringen Studierende in den Präsentationsszenarien mit größeren textuellen Anteilen auf den projizierten Folien deutlich bessere Wissensleistungen als in der nur durch Bilder und Grafiken visualisierten Präsentation.

Die schlechtesten Ergebnisse im Wissenstest erzielen die Rezipienten des ,Vortrags' mit 55\%. Der ,Vortrag' sowie die Präsentationsvarianten ,Bildfolien“ und ,Folienprojektion“ liegen dabei unter dem Durchschnittswert (siehe Abb. 6). Es fällt auf, dass zu den drei Szenarien mit den schlechtesten Ergebnissen die nicht multimodalen Formen ,Folienprojektion' und ,Vortrag' gehören. Deutlich besser scheint dabei die Vermittlung über den visuellen Kanal (,Folienprojektion') zu funktionieren; der Unterschied zwischen den Wissenstestergebnissen in den beiden Szenarien liegt bei $8 \%$. 
Die Wissensvermittlung mit der Unterstützung von projizierten Bildern, Grafiken etc. (,Bildfolien') bringt überraschenderweise sehr schlechte Ergebnisse (63\%). Bei einer näheren Betrachtung der Beantwortung einzelner Wissenstestfragen wird auch deutlich, dass die Probanden beispielsweise die Frage zu sog. Mashups ${ }^{17}$ in den Szenarien, in denen die Visualisierung des abstrakten Mashups-Beispiels verwendet wurde (siehe Abb. 3, Bildfolien), nicht besser beantworten. Die besten Antworten auf diese Frage geben die Rezipienten der Variante ,enge Verzahnung', in der sowohl ein Bullet Point mit einer Begriffserklärung zu Mashup als auch die Mashup-Visualisierung gezeigt wurde. Zusätzlich wurden in dieser Präsentationsvariante performative und sprachliche Verweismittel von dem Redner eingesetzt: Geste Richtung Projektionswand mit der mündlichen Erläuterung wie in der Abbildung visualisiert sowie Zeigegesten mit dem Pointer zum Zweck der Präzisierung der Grafikelemente.

Abschließend soll kurz auf die Memorierbarkeit der Inhalte eingegangen werden, die in der offenen Wissensfrage überprüft wurde. Die Frage nach den in der Präsentation genannten Konzernen, die semantische Technologien bereits einsetzen, wird von den Rezipienten der Szenarien ,Bildfolien" und ,Textfolien' am besten beantwortet. Erfolgreich sind somit zwei Varianten mit nicht animierten Folien, auf denen entweder (unter anderem) die Konzernnamen ausgeschrieben oder ausschließlich Konzernlogos gezeigt wurden, die die Konzernnamen beispielsweise als charakteristischen Schriftzug (etwa Yahoo!) beinhalten.

\subsection{Kommunikative Wirkung}

In dem Posttest-Fragebogen zur kommunikativen Wirkung der jeweiligen Präsentation wurden insgesamt maximal 14 Fragen gestellt. Die Anzahl der Fragen variierte, da beispielsweise nach der Präsentationsvariante ,Folienprojektion" keine Fragen zur Einschätzung des Rednerverhaltens und nach dem Szenario ,Vortrag' entsprechend keine Fragen zur Gestaltung der Powerpoint-Folien gestellt werden konnten.

Betrachtet man die einzelnen Fragen bzw. Aspekte der kommunikativen Wirkung und die pro Frage am besten bewerteten Szenarien, so schneidet die Variante, enge Verzahnung wesentlich besser als die anderen Präsentationsvarianten ab; sie erhält in sieben von vierzehn Aspekten die beste Bewertung (siehe Tab. 1). Insbesondere die modalitätsübergreifenden Aspekte, d. h. Fragen nach der Präsentationsstruktur und Informativität der Präsentation, nach dem Erkenntnisgewinn und dem Gesamteindruck, werden in diesem Szenario am besten bewertet. Das Szenario ,Identität' erreicht in zwei auf den Redner bezogenen Aspekten (Rednerverhalten, Rednersympathie) und in dem Aspekt der Themenat- 
Tabelle 1. Am besten bewertete Präsentationsszenarien nach Aspekten der kommunikativen Wirkung.

\begin{tabular}{ll}
\hline Szenario & Am besten bewertete Aspekte der kommunikativen Wirkung \\
\hline enge Verzahnung & $\begin{array}{l}\text { Foliengestaltung, Folienlesbarkeit, Logik der Folienabfolge, } \\
\text { Präsentationsstruktur, Informativität, } \\
\text { Erkenntnisgewinn, Gesamteindruck }\end{array}$ \\
Identität & Rednerverhalten, Rednersympathie, Thema interessant \\
Bildfolien & Bildereinsatz \\
Vortrag & Rednerkompetenz \\
Paraphrasierung & Einsatz der Präsentationstechnik \\
Folienprojektion & Textmenge pro Folie \\
\hline
\end{tabular}

traktivität die höchste Bewertung. Die Kompetenz des Redners wird im Szenario ,Vortrag' am besten gewertet.

Die Präsentation ,enge Verzahnung ' kann insgesamt als die kommunikativ erfolgreichste Variante angesehen werden. Sie hebt sich von den anderen Varianten ab. Wenn man sich das Vorkommen der einzelnen Szenarien unter den drei am besten bewerteten Szenarien pro Frage näher anschaut, erklimmt sie mit elf Vorkommen die Spitze des Rankings; es folgen „Bildfolien' (acht Vorkommen, darunter in den Fragen Informativität, Erkenntnisgewinn und Gesamteindruck), ,Identität (sieben Vorkommen, darunter jedoch in drei Fragen zum Redner/-verhalten, vgl. Tab. 1) und ,reduzierte Verzahnung' (sechs Vorkommen). In einem der erhobenen Aspekte schneidet ,enge Verzahnung' am schlechtesten ab, und zwar bezüglich der Rednersympathie. Den geringsten kommunikativen Erfolg verzeichnet das Szenario ,Folienprojektion'. Sieben Aspekte der kommunikativen Wirkung dieser Variante, darunter Informativität, Erkenntnisgewinn und Präsentationsstruktur, werden von den Rezipienten am schlechtesten beurteilt. Der klassische ,Vortrag" erhält in Bezug auf den Gesamteindruck und das Rednerverhalten die geringste Wertung. Die geringste Kompetenz wird dem Redner in der Variante mit stark paraphrasierenden Textfolien (,Paraphrasierung') zugesprochen. Die Foliengestaltung wird hier auch am niedrigsten gewertet.

Hinsichtlich des Zusammenhangs zwischen der subjektiven Bewertung der einzelnen Aspekte der kommunikativen Wirkung der Präsentationsvarianten und den Wissenstestergebnissen konnten lediglich zwei übergreifende Korrelationen ermittelt werden. Die Studierenden, die das Präsentationsthema als sehr interessant empfinden, sowie diejenigen, die die Präsentationsstruktur als sehr gut einschätzen, zeigen tendenziell bessere Leistungen im Wissenstest. 


\section{$5.3 \mathrm{Zu}$ Aspekten der performativen Dimension}

Aus Sicht der Rezipienten wird die Bedeutung einer Präsentation aus dem Gesamteindruck gebildet, den die in der sprachlichen und visuellen Modalität transportierten Inhalte und die Performanz des Präsentators ergeben. Die Frage nach dem Einfluss der performativen Dimension auf den Kommunikationserfolg wissenschaftlicher Präsentationen wurde bisher kaum zum Forschungsgegenstand gemacht. Die Befunde aus dem Gießener Experiment lassen sich im Hinblick auf diese Frage betrachten und können einige Hinweise zu ihrer Beantwortung geben. Für diesen Zweck können Ergebnisse der Posttest-Befragungen von drei Szenarien mit unterschiedlicher Ausprägung der performativen Modalität miteinander verglichen werden, d. h. des ,Vortrags ${ }^{6}$, der ,engen Verzahnung ${ }^{6}$ und der ,Folienprojektion". Die performative Dimension war dabei in der Präsentation ,enge Verzahnung' sehr deutlich ausgeprägt: Die sprachliche und visuelle Modalität wurden hier in hohem Maße durch performative deiktische Handlungen des Präsentators miteinander verknüpft. ,Vortrag' kennzeichnete sich lediglich durch die körperliche Präsenz des Redners, in der ,Folienprojektion“ fehlte der Redner gänzlich (vgl. Abschnitt 4.2).

Die kommunikative Wirkung der performativen Präsentationsvariante wird von den Rezipienten insgesamt sehr positiv bewertet, wie schon im Abschnitt 5.2 dargestellt; im Vergleich mit dem ,Vortrag ${ }^{6}$ und der ,Folienprojektion' schneidet sie am besten $a b-$ auch in Bezug auf die Effektivität der Wissensvermittlung (vgl. Abb. 6). In der Tab. 2 werden die Ergebnisse im Hinblick auf fünf ausgewählte Aspekte der kommunikativen Wirkung (Thema interessant, Informativität der jeweiligen Präsentationsform, Präsentationsstruktur, Erkenntnisgewinn und Gesamteindruck) in Form eines Szenarien-Rankings dargestellt. Anzumerken ist hierbei allerdings, dass die Unterschiede zwischen den ermittelten Mittelwerten sehr gering sind. Sie können also allenfalls erste Hinweise auf

Tabelle 2. Aspekte der kommunikativen Wirkung und Wissenstestergebnisse - Ranking dieser Präsentationsszenarien.

\begin{tabular}{llll}
\hline & Ranking & & \\
\cline { 2 - 4 } & $\mathbf{1 .}$ & $\mathbf{2 .}$ & $\mathbf{3 .}$ \\
\hline Thema interessant & Vortrag & enge Verzahnung & Projektion \\
Struktur & enge Verzahnung & Vortrag & Projektion \\
Informativität & enge Verzahnung & Vortrag & Projektion \\
Erkenntnisgewinn & enge Verzahnung & Vortrag & Projektion \\
Gesamteindruck & enge Verzahnung & Projektion & Vortrag \\
\hline Wissenstest & enge Verzahnung & Projektion & Vortrag \\
\hline
\end{tabular}


die Rolle der performativen Dimension geben. Die insgesamt bessere Bewertung des Kommunikationserfolgs des, Vortrags' im Vergleich zur ,Folienprojektion' lässt darauf schließen, dass allein die körperliche Präsenz eines Redners eine positive Wirkung zeigt. Der Redner in den Experimenten konnte ohne die PPT-Unterstützung beispielsweise auch für das Vortragsthema stärker interessieren.

In einigen freiwilligen Anmerkungen im Posttestfragebogen zum, Vortrag ${ }^{6}$ monieren die Rezipienten allerdings explizit die fehlende Unterstuitzung mit Präsentationstechnik etwa folgenderweise:

- „Bessere Qualität durch PPT-Folien und langsameren Redefluss könnte erreicht werden"

- „teilweise wäre eine Unterstützung des Vortrags mit Bildern hilfreich gewesen. Z. B. Suche von schlagwörtern mit Google im Vergleich zu einer Semantic-Anwendung"

- „keine Gliederung an der Wand oder vor den Zuhörern, es fällt schwer etwas zu verfolgen, wenn der rote Faden nicht ersichtlich ist“"

Folgende zwei Kommentare bringen die fehlende visuelle Modalität im ,Vortrag in Zusammenhang mit Verständnisschwierigkeiten:

- „Keine visuelle Unterstützung, das war schlecht! Mit Folien etc. hätte man sich die vielen neuen Begriffe viel besser einprägen und Zusammenhänge besser verstehen können. Sehr angenehme Sprechweise. $[\ldots]^{\text {" }}$

- „An sich war es ein guter Vortrag, aber als Powerpoint-verwöhnter Student ist es (besonders bei neuen Fachwörtern aus dem Englischen) sehr schwierig, die Informationsflut nur übers Ohr zu behalten."

Was die Wissensleistungen der Rezipienten angeht, so ist das gute Ergebnis in der Variante ,Folienprojektion' erstaunlich, wenn man bedenkt, dass in diesem Szenario die Rede fehlte und dementsprechend nicht alle Inhalte über den visuellen Kanal kommuniziert wurden.

\section{Interpretation der Ergebnisse}

Die im vorliegenden Beitrag präsentierten Befunde zur Rezeption wissenschaftlicher Präsentationen (siehe Abschnitt 5) geben empirisch begründete Hinweise, auf deren Basis die kommunikative Leistung wissenschaftlicher Präsentationen realistischer bewertet werden kann. Anhand der Detailbefunde lassen sich dabei Aussagen zur kommunikativen und kognitiven Wirkung von Präsentationen ableiten, die auch als Empfehlungen aufgefasst werden können (siehe unten). Sie stellen somit einen 
ersten Versuch in Richtung empirisch begründeter Präsentationsrhetorik dar. Nicht zuletzt relativieren sie die in der „Powerpoint ist böse“-Debatte vertretenen Positionen zu Auswirkungen der Präsentationssoftware auf Wissenschaft, Gesellschaft, Bildung und Sprache (vgl. Abschnitt 2) und bieten vielfältige Anknüpfungspunkte für die (empirische) Präsentationsforschung.

\section{Optimale Wissensvermittlung in wissenschaftlichen Präsentationen}

- Die simultane Vermittlung von gesprochenem und schriftlich projiziertem Text führt nicht zwangsläufig zu Cognitive Overload-Effekten. Die Redundanz von Rede und Textfolien kann insbesondere in kurzen Präsentationssequenzen zu einer höheren Behaltensleistung führen.

Die Redundanz gesprochener Sprache und projizierter Textelemente in multimodalen Präsentationen muss sich nicht negativ auf die Wissensleistungen der Rezipienten auswirken. Auch Paraphrasierungen der textuellen Folienelemente in der Rede führen nicht zwingend zur Beeinträchtigung der Aufnahmefähigkeit oder gar zu Überforderung der Rezipienten. Dies betrifft insbesondere kürzere Präsentationssequenzen.

- Die Verwendung von Bildern bzw. Visualisierungen auf den Präsentationsfolien unterstützt nicht per se die Memorierbarkeit und Verstehensprozesse.

Bilder, Illustrationen und schematische Darstellungen steigern in Kombination mit projizierten textuellen Erläuterungen die Behaltensleistung und tragen zur Verbesserung des Verständnisses bei. Der Bildüberlegenheitseffekt im Kontext wissenschaftlicher Präsentationen kann daher nicht (eindeutig) bestätigt werden.

- Gezielte Aufmerksamkeitsteuerung in Präsentationen durch Animationen oder durch sprachliche und performative Verweismittel des Redners effektiviert die Wissensvermittlung.

Insbesondere eine schrittweise Einblendung von textuellen Elementen auf projizierten Textfolien wirkte sich in Gießener Experimenten positiv auf die Wissensleistungen der Rezipienten aus. Bei sprachlichen Verweisen auf die Folien und Zeigegesten kann man ebenso von einer positiven Wirkung ausgehen. 


\section{Kommunikativer Erfolg wissenschaftlicher Präsentationen}

- Der Einsatz von Bildern und Grafiken zur visuellen Unterstützung der Rede steigert die positive subjektive Wahrnehmung der Kommunikationsqualität.

Obwohl der Bildüberlegenheitseffekt in den getesteten Präsentationen nicht bestätigt werden konnte, kann man von einer positiven kommunikativen Wirkung sprechen, die funktional eingesetzte Bild- und Grafikelemente auf die Rezipienten haben. Insbesondere in Bezug auf modalitätsübergreifende Aspekte wie Informativität, Erkenntnisgewinn und Gesamteindruck erhalten entsprechende Präsentationsszenarien (,enge Verzahnung" und ,Bildfolien') von den Rezipienten eine sehr gute Bewertung.

- Eine positive kommunikative Wirkung multimodaler Präsentationen hängt mit dem Rednerverhalten und seiner rhetorischen und performativen Wirkung zusammen. Sprachliche und performative Verweismittel des Redners können die wahrgenommene Kommunikationsqualität erhöhen.

Der Redner und sein Verhalten spielen eine nicht zu unterschätzende Rolle für den Kommunikationserfolg der Präsentationen. Als eines der Indizien dafür kann man bereits die negative Resonanz der Rezipienten in den Gießener Experimenten zur ,Folienprojektion' ohne Redner ansehen. Die Rolle der performativen Dimension und des Redners begründet aber vor allem das durchweg positive Feedback der Rezipienten zum performativ inszenierten Szenario, enge Verzahnung".

\section{Erfolgreiche Präsentationen - Zur Verzahnung der Modalitäten}

- Eine erfolgreiche Präsentation erfordert einen kompetenten Autor, Regisseur, Bühnenbildner, Redner und Darsteller zugleich. Darüber hinaus erfordert sie auch einen reflektierten Kohärenz-Manager, der modalitätsübergreifende Zusammenhänge gezielt konstituiert und organisiert.

Das Verständnis und die Überzeugungskraft wissenschaftlicher Erkenntnisse können in Präsentationen durch das Verhalten des Präsentators deutlich beeinflusst werden. Allein die körperliche Präsenz des Redners in Präsentationen - eine (gute) Vorbereitung bzw. Inszenierung vorausgesetzt - ist für ihren Erfolg aber nicht ausreichend. Erfolgreiche Präsentationen bedürfen einer engen Verzahnung des Gesagten mit dem Gezeigten durch ein gezieltes Kohärenz-Management mittels Rekurrenz und 
mündlicher und performativer Verweise (wie Zeigegesten oder Körperstellung) des Redners. Diese Mittel der Verzahnung von Rede und Folienprojektion erhöhen nicht nur die kommunikative Wirkung, sondern effektivieren beim gezielten und reflektierten Einsatz die Informationsvermittlung als solche.

\section{Fazit}

(Wie) Kann man in der Wissenschaft erfolgreich präsentieren? Trotz aller bekannten Unzulänglichkeiten der Präsentationssoftware Powerpoint erhöht ihr reflektierter Einsatz den kommunikativen Erfolg und - auf jeden Fall kurzfristig - die Effektivität der Informationsvermittlung.

Im Lichte der Befunde aus den Gießener Experimenten zur Präsentationsqualität erscheint daher die medien- und kulturpessimistische Kritik an (wissenschaftlichen) Präsentationen als oberflächlich. Die visuelle Unterstützung des Vortrags mit Präsentationsfolien ist durchaus sinnvoll und für den Zweck einer effektiven Informationsvermittlung vorteilhaft. Alle sieben getesteten Szenarien mit Präsentationsfolien haben wesentlich mehr Erfolg bei den Rezipienten als der klassische Vortrag. Aus kommunikativer Sicht haben Präsentationen als Kommunikationsform in der modernen Wissenschaft ihre Berechtigung also nicht alleine als Antwort auf den allgegenwärtigen Bedarf nach schnellem und effizientem Informationsaustausch. In der Kommunikationspraxis der Hochschule werden sie als eine gängige und durchaus erfolgreiche Kommunikationsform gar erwartet, was auch auf ein verändertes Rezeptionsverhalten hinweist (siehe Kommentare der Rezipienten im Abschnitt 5.3).

\section{Anmerkungen}

1. Die Ausdrücke (wissenschaftliche) Präsentationen sowie (wissenschaftliche) Präsentationen mit Unterstützung von Präsentationssoftware werden im Beitrag synonym verwendet (siehe Lobin 2009a: 16).

2. Die Experimente wurden im Rahmen des Projekts „Wissenschaftliche Präsentationen" innerhalb des Forschungsverbundes „Interne Wissenschaftskommunikation über digitale Medien“ an der Universität Gießen im Sommer 2010 durchgeführt (siehe www.wissenschaftskommunikation.info). Im Projekt kooperierte das Zentrum für Medien und Interaktivität der Universität Gießen mit der Medienwissenschaft der Universität Trier.

3. Es sei an dieser Stelle insbesondere auf die Überblicksdarstellungen in Mertens (2004) und Bieber (2009) hingewiesen.

4. Die Ergebnisse beruhen auf einer von Betül Özsarigöl durchgeführten Auswertung von dreißig Ratgebern zu Präsentationen mit Softwareunterstützung.

5. „Selbst wer Regeln zur Schriftgröße, -farbe und zum Präsentationsmodus befolgt, hat nicht gleichzeitig die Garantie, dass es eine gute Präsentation wird“, konstatiert Weil (2009: 25). 
6. Hinsichtlich der von Pflüger (2009) geforderten „einfachen Handhabung“ von Präsentationssoftware sind darüber hinaus Untersuchungen zu Usability von Powerpoint von großem Interesse, wie beispielsweise Thielsch und Förster (2007).

7. An dieser Stelle sei auf die pädagogischen Experimente zur „Effektstärke von dynamischen Präsentationen" hingewiesen, die an der Universität Rostock von Studierenden durchgeführt wurden. Die Studierenden kommen dabei zu dem Schluss, Animationen würden das Arbeitsgedächtnis unnötig belasten und Lerneffekte verhindern (siehe Nieke 2011).

8. Die Datenerhebung zum Zweck der Korpusbildung wurde vom Juni 2008 bis Februar 2009 von den Medienwissenschaftlern der Universität Trier vorgenommen. Sie umfasste unter anderem Ton- und Videoaufzeichnungen des Präsentators an einem (ggf. angedeuteten) Rednerpult samt der Fläche für die Projektion von Präsentationsfolien. In nahezu allen aufgezeichneten Vorträgen wurde die Präsentationssoftware Powerpoint eingesetzt. Übersicht der aufgezeichneten Präsentationen siehe Bucher et al. (2010: 406).

9. In anderer Qualität und Ausprägung kommt Multimodalität bereits in früheren Vortragsformen vor.

10. Aufführung ist eine Präsentationsphase - neben der Inszenierung im Sinne der Planung der Aufführung und der Archivierung (siehe Lobin 2007: 68 f.). Diese Präsentationsphasen können in allen drei Modalitäten unterschieden werden.

11. Die Grundlage der Folienerfassung in den Präsentationen des Korpus bildete eine empirisch fundierte Folientypologie, siehe Lobin et al. (2010: 361).

12. Dieser Befund stimmt somit nicht mit der vorherrschenden, allerdings bisher empirisch nicht gesicherten Meinung überein, dass beim Präsentieren die „Naturwissenschaften [...] zum Bildlichen, Geisteswissenschaften zum Text [neigen]", siehe Günther und Knoblauch (2007: 62).

13. Bucher et al. (2010) zählen das Textdesign der Folien zu Elementen der Performanz wissenschaftlicher Präsentationen (ebd., 376).

14. Eine Ausnahme bildete die Präsentationsvariante ,Folienprojektion", siehe Überblick über die Szenarien.

15. Die Mittelwerte der Einschätzung in den einzelnen Szenarien lagen beim thematischen Vorwissen zwischen den Schulnoten 4 bis 4,8 und bei den PowerpointKenntnissen zwischen den Noten 2 bis 2,6.

16. Die Standardabweichung liegt durchschnittlich zwischen 0,2 bis 0,3 Punkten.

17. Im Wissenstest sollten die Testpersonen zutreffende Aussagen ankreuzen, die in der Präsentation zu Mashups gemacht wurden. Drei Antwortvorgaben standen dabei zur Verfügung, u. a. die (richtige) Aussage In Mashup-Anwendungen werden Daten aus unterschiedlichen Quellen integriert.

\section{Literatur}

Adams, Catherine. 2008. PowerPoint, Denkgewohnheiten, Unterrichtskultur. Erziehungswissenschaft. 19. 7-31.

Antos, Gerd. 2009. Semiotik der Text-Performanz. In Angelika Linke \& Helmuth Feilke (Hrsg.), Oberfläche und Performanz. Untersuchungen zur Sprache als dynamischer Gestalt, 407-427. Tübingen.

Bartsch, Robert A. \& Cobern, Kristi M. 2003. Effectiveness of PowerPoint presentations in lectures. Computers \& Education. 41. 77-86.

Becker, Lisa. 2005. Publikumsprügelei mit Powerpoint. Frankfurter Allgemeine Zeitung. 22. 01.2005. 53. http://www.faz.net/s/

RubB1E10A8367E8446897468EDAA6EA0504/Doc 
EAAA2F869666D4ACD9CBCAE0094E7884B ATp1 Ecommon $\sim$ Scontent.html (zuletzt geprüft: 17.01.2012).

Bieber, Christoph. 2009. Ist Powerpoint böse? Öffentlich Debatten in den USA und Deutschland. In Wolfgang Coy \& Claus Pias (Hrsg.), Power Point. Macht und Einfluss eines Präsentationsprogramms, 125-145. Frankfurt am Main.

Brinkschulte, Melanie. 2007. Lokaldeiktische Prozeduren als Mittler zwischen Rede und Powerpoint-Präsentation in Vorlesungen. In Bernt Schnettler \& Hubert Knoblauch (Hrsg.), Powerpoint-Präsentationen: Neue Formen der gesellschaftlichen Kommunikation von Wissen, 104-116. Konstanz.

Bucher, Hans-Jürgen. 2007. Textdesign und Multimodalität. Zur Semantik und Pragmatik medialer Gestaltungsformen. In: Kersten Sven Roth \& Jürgen Spitzmüller (Hrsg.), Textdesign und Textwirkung in der massenmedialen Kommunikation, 35-64. Konstanz.

Bucher, Hans-Jürgen, Niemann, Philipp \& Krieg, Martin. 2010. Die wissenschaftliche Präsentation als multimodale Kommunikationsform. Empirische Befunde zu Rezeption und Verständlichkeit von Powerpoint-Präsentationen. In Hans-Jürgen Bucher, Thomas Gloning \& Katrin Lehnen (Hrsg.), Neue Medien - neue Formate. Ausdifferenzierung und Konvergenz in der Medienkommunikation, 375-406. Frankfurt am Main.

Coy, Wolfganf \& Pias, Claus (Hrsg.). 2009. PowerPoint. Macht und Einfluss eines Präsentationsprogramms. Frankfurt am Main.

Dambeck, Holger. 2006. Maulwurf-Pellets für Verwaltungsnerds. Spiegel. 01. 02. 2006. http://Www.spiegel.de/netzwelt/web/0,1518,398488,00.html (zuletzt geprüft: 17.01 . 2012).

Danz, Gerriet. 2010. Neu präsentieren. Begeistern und überzeugen mit den Erfolgsmethoden der Werbung. Frankfurt am Main.

Earnest, William James. 2006. Developing strategies to evaluate the effective use of electronic presentation software in communication education. The University of Texas at Austin dissertation http://hdl.handle.net/2152/257 (zuletzt geprüft: 17. 01. 2012).

Fischer-Lichte, Erika (Hrsg.). 2005. Diskurse des Theatralen. Tübingen.

Frey, Barbara \& Birnbaum, David. 2002. Learners' Perceptions on the Value of PowerPoint in Lectures. Educational Resources Information Center. $1-9$.

Fricke, Ellen. 2008. PowerPoint und Overhead: Mediale und konzeptuelle Bedingungen des mündlichen Vortrags aus deixistheoretischer Perspektive. Zeitschrift für Semiotik. 30.151-173.

Grabowski, Joachim. 2003. Kongressvorträge und Medieneinsatz: ein Plädoyer für Professionalität. Zeitschrift für Angewandte Linguistik (39). 53-73.

Grabowski, Joachim. 2008. Aufmerksamkeitslenkung bei foliengestützten Vorträgen: Drei Aspekte der räumlichen Koordination und die Schwierigkeit von PowerPointPräsentationen. Zeitschrift für Semiotik. 30. 137-150.

Günther, Susanne \& Knoblauch, Hubert. 2007. Wissenschaftliche Diskursgattungen PowerPoint et al. In Peter Auer \& Harald Baßler (Hrsg.), Reden und schreiben in der Wissenschaft, 52-65. Frankfurt am Main.

Joffe, Josef. 2007. An die Wand geworfen. Warum PowerPoint-Präsentationen und Marketing-Jargon Sprache und Geist beschädigen. Die Zeit. 26.07.2007. http:// www.zeit.de/2007/31/Deutsch-Speak (zuletzt geprüft: 17.01.2012).

Keller, Julia. 2003. Is PowerPoint The Devil? Chicago Tribune. 22. 01. 2003. http://faculty. winthrop.edu/kosterj/WRIT465/management/juliakellerl.htm (zuletzt geprüft: 17. 01.2012).

Knape, Joachim. 2007. Powerpoint in rhetoriktheoretischer Sicht. In Bernt Schnettler \& Hubert Knoblauch (Hrsg.), Powerpoint-Präsentationen: Neue Formen der gesellschaftlichen Kommunikation von Wissen, 53-66. Konstanz. 
Knoblauch, Hubert. 2007. Die Performanz des Wissens. Zeigen und Wissen in Powerpoint-Präsentationen. In Bernt Schnettler \& Hubert Knoblauch (Hrsg.), PowerpointPräsentationen: Neue Formen der gesellschaftichen Kommunikation von Wissen, 117 137. Konstanz.

Knoblauch, Hubert. 2009. Wissen Life: Sitzordnung, Performanz und PowerPoint. In Herbert Willems \& Günter Burkart (Hrsg.), Theatralisierung der Gesellschaft, 221237. Wiesbaden.

König, Anika. 2007. Zur Güte von Präsentationen - normative vs. Ethnokategorien für angemessene Powerpoint-Präsentationen. In Bernt Schnettler \& Hubert Knoblauch (Hrsg.), Powerpoint-Präsentationen: Neue Formen der gesellschaftlichen Kommunikation von Wissen, 207-223. Konstanz.

Lobin, Henning. 2007. Textsorte, Wissenschaftliche Präsentation" - Tex tlinguistische Bemerkungen zu einer komplexen Kommunikationsform. In Bernt Schnettler \& Hubert Knoblauch (Hrsg.), Powerpoint-Präsentationen: Neue Formen der gesellschaftlichen Kommunikation von Wissen, 67-82. Konstanz.

Lobin, Henning. 2009a. Inszeniertes Reden auf der Medienbiihne. Zur Linguistik und Rhetorik der wissenschaftlichen Präsentation. Frankfurt am Main, New York.

Lobin, Henning. 2009b. Rhetorik ist nicht alles. Powerpoint geht über die Rede hinaus. Sïddeutsche Zeitung. 8. 12. 2009.

Lobin, Henning, Dynkowska, Malgorzata \&Özsarigöl, Betül. 2010. Formen und Muster der Multimodalität in wissenschaftlichen Präsentationen. In Hans-Jürgen Bucher, Thomas Gloning \& Katrin Lehnen (Hrsg.), Neue Medien - neue Formate. Ausdifferenzierung und Konvergenz in der Medienkommunikation, 357-374. Frankfurt am Main.

Lowry, Roy B. 1999. Electronic Presentation of Lectures - Effect Upon Student Performance. University Chemistry Education. 3. 18-21.

Mahar, Stephen, Yaylacicegi, Ulku \& Janicki, Thomas. 2009. The dark side of custom animation. International Journal of Innovation and Learning, 6 (6). 581-592.

Mertens, Mathias. 2004. Die Debatte um PowerPoint (Dossier). http://www.zmi.unigiessen.de/home/publikation-powerpoint.html (zuletzt geprüft: 17.01. 2012).

Müller-Prove, Matthias. 2009. Slideware. Kommunikationsmedium zwischen Redner und Publikum. In Wolfgang Coy \& Claus Pias (Hrsg.), PowerPoint. Macht und Einfluss eines Präsentationsprogramms, 45-62. Frankfurt am Main.

Nieke, Wolfgang. 2011. Was nützen dynamische Animationen für die Unterstützung von Präsentationen in Unterrichts- und Unterweisungssituationen? Elektronisches Dokument. Rostock. http://rosdok.uni-rostock.de/file/rosdok_derivate_000000004699/ Nieke-Dynamische_Animationen-2011.pdf (zuletzt geprüft: 25. 01. 2012).

Niemann, Philipp \& Krieg, Martin. 2011. Von der Bleiwüste bis zur Diashow: Zur Rezeption zentraler Formen wissenschaftlicher Präsentationen. Zeitschrift für Angewandte Linguistik, 54 (1). 111-143.

Peters, Sibylle. 2007. Über Ablenkung in der Präsentation von Wissen. Freier Vortrag, Lichtbild-Vortrag und Powerpoint-Präsentation - ein Vergleich. In Bernt Schnettler \& Hubert Knoblauch (Hrsg.), Powerpoint-Präsentationen: Neue Formen der gesellschaftlichen Kommunikation von Wissen, 37-52. Konstanz.

Peters, Sibylle. 2008. Die Präsentation der Präsentation. Im Bilde Sein in Zeiten von Powerpoint. In Gottfried Boehm, Birgit Mersmann \& Christian Spies (Hrsg.), Movens Bild. Zwischen Evidenz und Affekt, 367-382. München.

Peters, Sibylle. 2009. Wir Schauspieler. Wie Powerpoint Wissen verändert. Süddeutsche Zeitung, 28. 12. 2009. Nr. 298, 13.

Peters, Sibylle. 2011. Der Vortrag als Performance. Bielefeld.

Pflüger, Jörg. 2009. Auf den Punkt gebracht. Prolegomena zu einer Rhetorik der Präsentation. In Wolfgang Coy \& Claus Pias (Hrsg.), PowerPoint. Macht und Einfluss eines Präsentationsprogramms, 146-216. Frankfurt am Main. 
Pötzsch, Frederik S. 2007. Der Vollzug der Evidenz. Zur Ikonographie und Pragmatik von Powerpoint-Folien. In Bernt Schnettler \& Hubert Knoblauch (Hrsg.), Powerpoint-Präsentationen: Neue Formen der gesellschaftlichen Kommunikation von Wissen, 83-103. Konstanz.

Rankin, Elizabeth L. \& Hoaas, David J. 2001. The Use of PowerPoint and Student Performance. Atlantic Economic Journal. (29). 113.

Rebensburg, Klaus. 2009. Worst Practice mit PowerPoint. Von Kraftpunkten, Kraftlosigkeit und Katastrophen der Informatik. In Wolfgang Coy \& Claus Pias (Hrsg.), Power Point. Macht und Einfluss eines Präsentationsprogramms, 87-124. Frankfurt am Main.

Ross, Alexander. 2008. Folienschlacht mit Zischgeräusch. Handelsblatt. 01. 03.2008. http://www.handelsblatt.com/karriere/nachrichten/folienschlacht-mitzischgeraeusch/2928522.html?p2928522=all (zuletzt geprüft: 11. 01. 2012).

Schlaffer, Heinz. 2004. Menetekel. Frankfurter Rundschau. 27. 01. 2004. http://sform. bildung.hessen.de/gymnasium/skii/fuenftes_pf/news_pruef/1102257090 (zuletzt geprüft: 17.01 .2012 ).

Schnettler, Bernt. 2007. Zur Orchestrierung von Listen. Eine Videoperformanzanalyse. In Bernt Schnettler \& Hubert Knoblauch (Hrsg.), Powerpoint-Präsentationen: Neue Formen der gesellschaftlichen Kommunikation von Wissen, 139-159. Konstanz.

Schnettler, Bernt, Knoblauch, Hubert \& Pötzsch, Frederik S. 2007. Die PowerpointPräsentation. Zur Performanz technisierter mündlicher Gattungen in der Wissensgesellschaft. In Bernt Schnettler \& Hubert Knoblauch (Hrsg.), Powerpoint-Präsentationen: Neue Formen der gesellschaftlichen Kommunikation von Wissen, 9-34. Konstanz.

Schnettler, Bernt \& Knoblauch, Hubert (Hrsg.). 2007. Powerpoint-Präsentationen: Neue Formen der gesellschaftlichen Kommunikation von Wissen. Konstanz.

Shwom, Barbara L. \& Keller, Karl P. 2003. „The Great Man Has Spoken. Now What Do I Do?" A Response to Edward Tufte's „The Cognitive Style of PowerPoint”. Communication Insight. (1). 1-16.

Steinfeld, Thomas. 2009. Ist PowerPoint gefährlich? Süddeutsche Zeitung. 30. 11. 2009. $\mathrm{http} / / \mathrm{www}$.sueddeutsche.de/kul tur/unbehagen-an-praesentationsprogramm-istpowerpoint-gefaehrlich-1.127616 (zuletzt geprüft: 11. 01. 2012).

Stoll, Clifford. 2002. LogOut. Warum Computer nichts im Klassenzimmer zu suchen haben und andere High-Tech-Ketzereien. Frankfurt am Main.

Sweller, John (Hrsg.). 2002. Visualisation and Instructional Design. International Workshop on Dynamic Visualizations and Learning. Tübingen.

Szabo, Attila \& Hastings, Nigel. 2000. Using IT in the undergraduate classroom: should we replace the blackboard with PowerPoint. Computers \& Education. (35). 175187.

Thielsch, Meinald \& Förster, Natalie. 2007. Präsentationssoftware: Nutzung und funktionale Anforderungen. In Kerstin Röse \& Henning Brau (Hrsg.), Usability Professionals, $1-7$. Stuttgart.

Tufte, Edward R. 2003a. PowerPoint Is Evil. Power Corrupts. PowerPoint Corrupts Absolutely. Wired Magazin. 11 (9).

Tufte, Edward R. 2003b. The cognitive style of PowerPoint. Pitching out corrupts within. Cheshire, Connecticut.

Weil, Markus. 2009. „Powerpointismus“ und die Rolle der Bilder in Präsentationen. Education Permanente. 2. 24-25.

Yates, JoAnne \& Orlikowski, Wanda. 2007. Die Powerpoint-Präsentation und ihre Abkömmlinge. Wie Gattungen das kommunikative Handeln in Organisationen prägen. In Bernt Schnettler \& Hubert Knoblauch (Hrsg.), Powerpoint-Präsentationen: Neue Formen der gesellschaftichen Kommunikation von Wissen, 225-248. Konstanz. 
Young, Jeffrey R. 2004. When Good Technology Means Bad Teaching. Chronicle of Higher Education 51 (12), A31.

Zillien, Nicole. 2005. „PowerPoint makes you dumb“. Ein Klärungsversuch mit Hilfe der Theorie der Strukturierung. In Michael Jäckel \& Manfred Mai, (Hrsg.), OnlineVergesellschaftung. Mediensoziologische Perspektiven auf neue Kommunikationstechnologien, 155-174. Wiesbaden.

Zillien, Nicole. 2006. „Nächste Folie bitte!“ Der Einsatz von Präsentationsprogrammen zur Wissensvermittlung und Wissensbewahrung. In Peter H. Ohly (Hrsg.). Wissensorganisation und Verantwortung. Gesellschaftliche, ökonomische und technische Aspekte. Proceedings der 9. Tagung der Deutschen Sektion der Internationalen Gesellschaft für Wissensorganisation Duisburg, 5.-7. November 2004, 159-168. Würzburg. 